\title{
Influences of cancer symptom knowledge, beliefs and barriers on cancer symptom presentation in relation to socioeconomic deprivation: a systematic review
}

\author{
Grace M. McCutchan*, Fiona Wood, Adrian Edwards, Rebecca Richards and Kate E. Brain
}

\begin{abstract}
Background: People from lower socioeconomic groups have worse survival outcomes for cancer, which in part reflects later-stage disease at diagnosis. The mechanisms underlying delayed cancer symptom presentation in lower socioeconomic groups are not well understood.

Methods: Systematic review of studies of actual or anticipated symptom presentation across all tumour sites. Included studies measured socioeconomic group, symptom presentation and one or more of the following variables: cancer symptom knowledge, beliefs about cancer, barriers/facilitators to symptom presentation.

Results: A total of 60 studies was included. Symptom knowledge overall was lowest and actual presentation time was longest in lower socioeconomic groups. Knowledge for specific symptoms such as lumps and bleeding was good and encouraged timely symptom presentation, in contrast to non-specific symptoms which were not well recognised. The combination of fearful and fatalistic beliefs was typically associated with later presentation, especially in lower socioeconomic groups. Emotional barriers such as 'worry what the doctor might find' were more frequently reported in lower socioeconomic groups, and there was evidence to suggest that disclosing symptoms to family/friends could help or hinder early presentation.

Conclusions: Poor symptom knowledge, fearful and fatalistic beliefs about cancer, and emotional barriers combine to prolong symptom presentation among lower socioeconomic groups. Targeted interventions should utilise social networks to improve knowledge of non-specific symptoms, challenge negative beliefs and encourage help-seeking, in order to reduce avoidable delays and minimise socioeconomic group inequalities.
\end{abstract}

Keywords: Patient delay, Symptom knowledge, Cancer beliefs, Barriers to symptom presentation, Socioeconomic status

\section{Background}

Socioeconomic inequalities in cancer survival outcomes exist, but the reasons for this are not fully understood [1-3]. Survival differences are likely to reflect later-stage disease at diagnosis $[2,4,5]$ partly as a consequence of delayed cancer symptom presentation in people from lower socioeconomic groups [6]. By eradicating socioeconomic inequalities at stage of diagnosis, it is estimated that 5600 patients in the UK annually could be diagnosed with earlier stage disease [7], and that $11 \%$ of

\footnotetext{
* Correspondence: mccutchangm@cardiff.ac.uk

Institute of Primary Care and Public Health, School of Medicine, Cardiff University, Neuadd Meirionnydd, Heath Park, Cardiff CF14 4YS, UK
}

deaths from cancer could be avoided if three-year survival in lower socioeconomic groups matched that in higher socioeconomic groups [1].

'Patient delay' is defined as the time between discovery of a cancer symptom and the initial visit to a healthcare professional. It accounts for the greatest proportion of delay time in the pathway from symptom discovery to the start of cancer treatment [8-10] and has been associated with socioeconomic deprivation [6]. Patient delay has been conceptualised in Walter et al.'s Model of Pathways to Treatment, with various stages involving an 'appraisal interval' during which the individual detects a bodily change, and a 'help seeking interval' 
in which the individual decides to seek medical help (see Fig. 1 [11]). Evidence suggests that knowledge of cancer symptoms is important during the appraisal stage, with potential misattribution of symptoms attenuating the decision to present [12, 13]. Beliefs about cancer are considered to be important in both the appraisal and help-seeking stages, where emotions such as fear might influence interpretation of symptoms [12] and the decision to seek medical help [6, 14-17]. Barriers such as competing life events and ease of getting a medical appointment are thought to delay symptom presentation during the help-seeking interval [11].

The contribution of socioeconomic and other demographic factors to delayed presentation has been highlighted in the Model of Pathways to Treatment, and more recently in the updated National Awareness and Early Diagnosis Initiative (NAEDI) framework designed to conceptualise the route from public awareness and beliefs about cancer to cancer survival outcomes ([18]). Empirical evidence supports associations between lower socioeconomic group and poor cancer symptom knowledge [19], fearful and fatalistic beliefs about cancer [20] and emotional barriers such as worry about what the doctor may find [19]. These findings help to explain why people from lower socioeconomic groups tend to present with more advanced stage cancers, and hence have worse survival outcomes [1-5]. However, a more detailed understanding of psychosocial influences on the relationship between socioeconomic deprivation and cancer symptom presentation is essential to developing behavioural interventions designed to promote timely presentation and reduce socioeconomic inequalities in cancer outcomes.

Attempts to understand why people might delay seeking medical help for cancer symptoms have examined actual or anticipated symptom presentation behaviour, exploring perceived barriers to symptom presentation. Prospective study designs are difficult due to follow-up of a large sample, so studies frequently use retrospectively recalled or hypothetically anticipated symptom study designs. Previous reviews have focused on tumour site-specific delay factors $[15,16,21]$ or common cancers only [6], or have been restricted to qualitative studies [17] and patients with cancer [6, 16, 17]. The purpose of the current systematic review was to explore how knowledge, beliefs and barriers/facilitators to symptom presentation affect actual or anticipated cancer symptom presentation in relation to socioeconomic group and across all tumour sites.

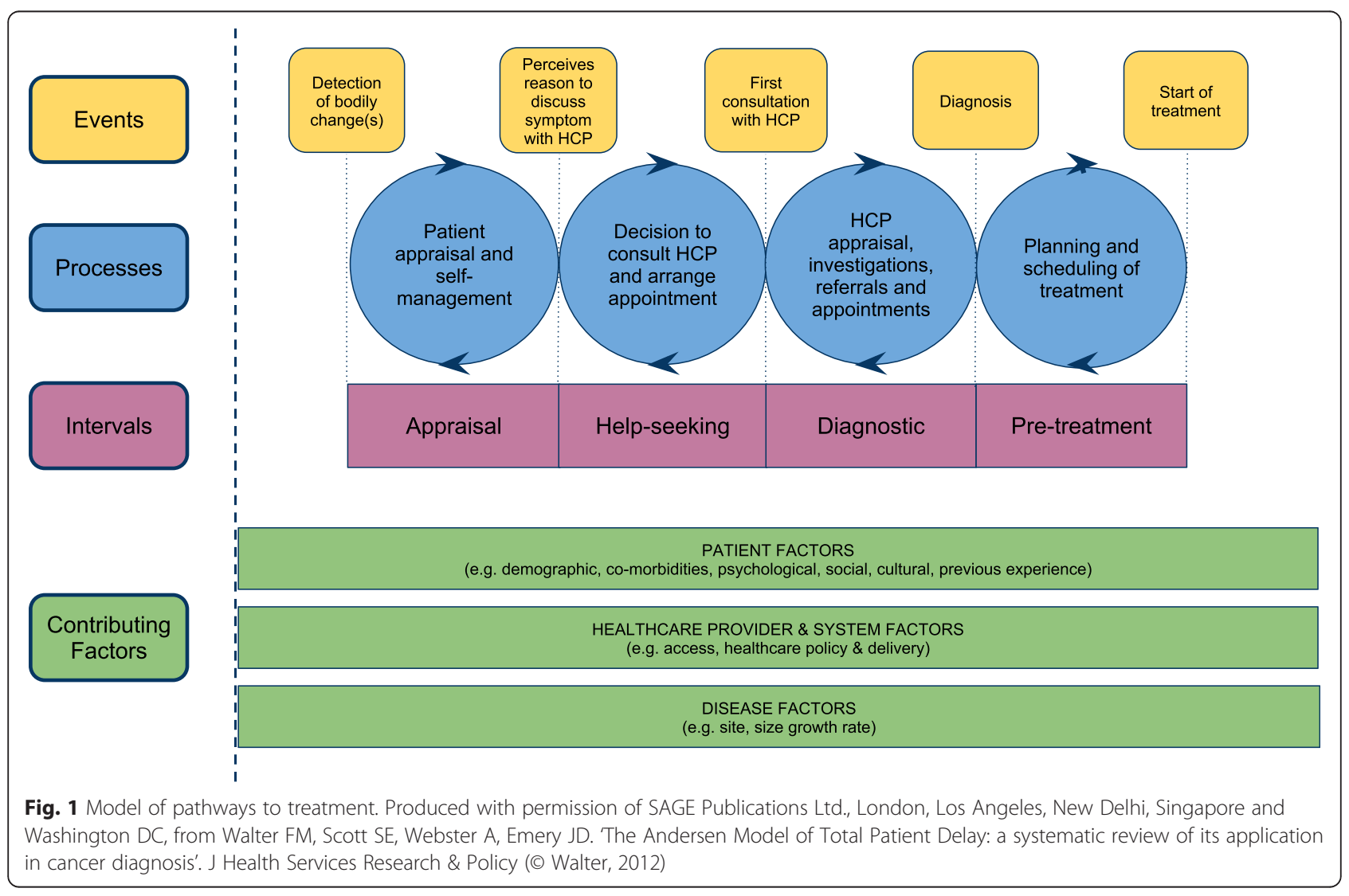




\section{Method}

Identification of included studies followed the PRISMA guidelines [22]. The protocol was registered on PROSPERO (CRD42014013220 [23]) and is available on the NIHR HTA programme website (www.hta.ac.uk). At all stages of the search, data extraction and quality appraisal, $10 \%$ of studies were double checked for consistency by a second member of the research team (RR). All discrepancies were resolved through discussion.

\section{Search strategy}

The literature was searched up to July 2015 on the electronic databases of MEDLINE, PsychINFO, EMBASE and CINAHL. The de-duplicate function was used on Ovid and CINAHL before reviewing abstracts. Manual searches of reference lists of included studies were performed. A SPIDER (Sample, Phenomenon of Interest, Design, Evaluation, Research type) search strategy tool was used for retrieval of studies (see Additional file 1: Appendix 1 [24]). Databases were searched using terms relating to symptom presentation, cancer symptom knowledge, beliefs about cancer, perceived barriers and facilitators to symptom presentation (see Additional file 1: Appendix 1).

\section{Inclusion criteria}

Publications that measured and reported data for symptom presentation and socioeconomic group were included. 'Symptom presentation' was defined as actual symptom presentation (retrospectively recalled) or anticipated symptom presentation (hypothetically estimated) measured as continuous (time to presentation) or binary (did/did not present) variables. 'Socioeconomic group' was defined in terms of individual level socioeconomic indicators including education, income, home/car ownership, occupation and employment, and/or area-level indicators based on postcode. In addition, publications were included if they measured and reported one or more of the following domains of interest:

- 'Knowledge': studies which assessed knowledge for the symptoms of cancer through recall e.g. 'What symptoms of cancer can you list?' or recognition methods e.g. "Which of these are symptoms of cancer?', or through retrospective recall of symptom interpretation at the time of symptom discovery.

- 'Beliefs': studies which explored any positive (e.g. beliefs about the benefits of early diagnosis and curability) or negative (e.g. fear and fatalism) beliefs surrounding cancer.

- 'Perceived barriers/facilitators': studies which assessed any anticipated or actual barriers or facilitators to symptom presentation.
There were no restrictions on date of publication or study methodology. Only English language studies from high income countries as classified by Organisation for Economic Co-operation and Development (OECD) membership (OECD, 2014 [25]) were included.

\section{Exclusion criteria}

Studies that did not measure and report symptom presentation, socioeconomic group and one or more of the domains of interest were excluded. Studies not relating to cancer, and those examining screening behaviour, selfexamination behaviour, efficacy of interventions, genetic risk, healthcare professionals' perspective, cancer prevention, treatments for cancer or living with cancer and studies involving children were excluded. Studies from low/middle income countries, not written in English, review papers or conference abstracts were excluded (Fig. 2).

\section{Data extraction and synthesis}

Data were extracted onto a template using the following headings: method, sample characteristics, tumour site, symptom presentation, knowledge, beliefs, perceived barriers/facilitators and socioeconomic group measure. A meta-analysis was precluded due to the heterogeneity of included studies and a narrative synthesis was performed [26].

\section{Critical appraisal}

The methodological quality of all included studies was examined using the Critical Appraisal Skills Programme tool (CASP, 2014 [27]) appropriate for the study design. Quality was assessed according to each domain on the CASP checklists: rationale of study, methodology, design, recruitment, data collection, data analysis, ethical issues, reporting of findings and contribution to research. Overall quality was categorised as good, medium or poor.

\section{Results}

The search returned a total of 1536 studies after 810 duplicates had been removed. A total of 1309 studies was excluded based on title and abstract, leaving 227 studies to be read in full. A total of 60 studies met the inclusion criteria (see Fig. 2). Eleven of these studies were found through hand searching reference lists.

Included studies employed qualitative methods $(n=15)$, quantitative methods $(n=42)$ and mixed methods $(n=3)$. Quality of studies was good $(n=18)$, medium $(n=37)$ and poor $(n=5)$. Limitations of lower quality studies included measuring but not reporting socioeconomic group differences for all outcome measures, leaving a long period of time between cancer diagnosis and participation in the study and recruitment of samples biased towards higher socioeconomic groups. The overall combined percentage 


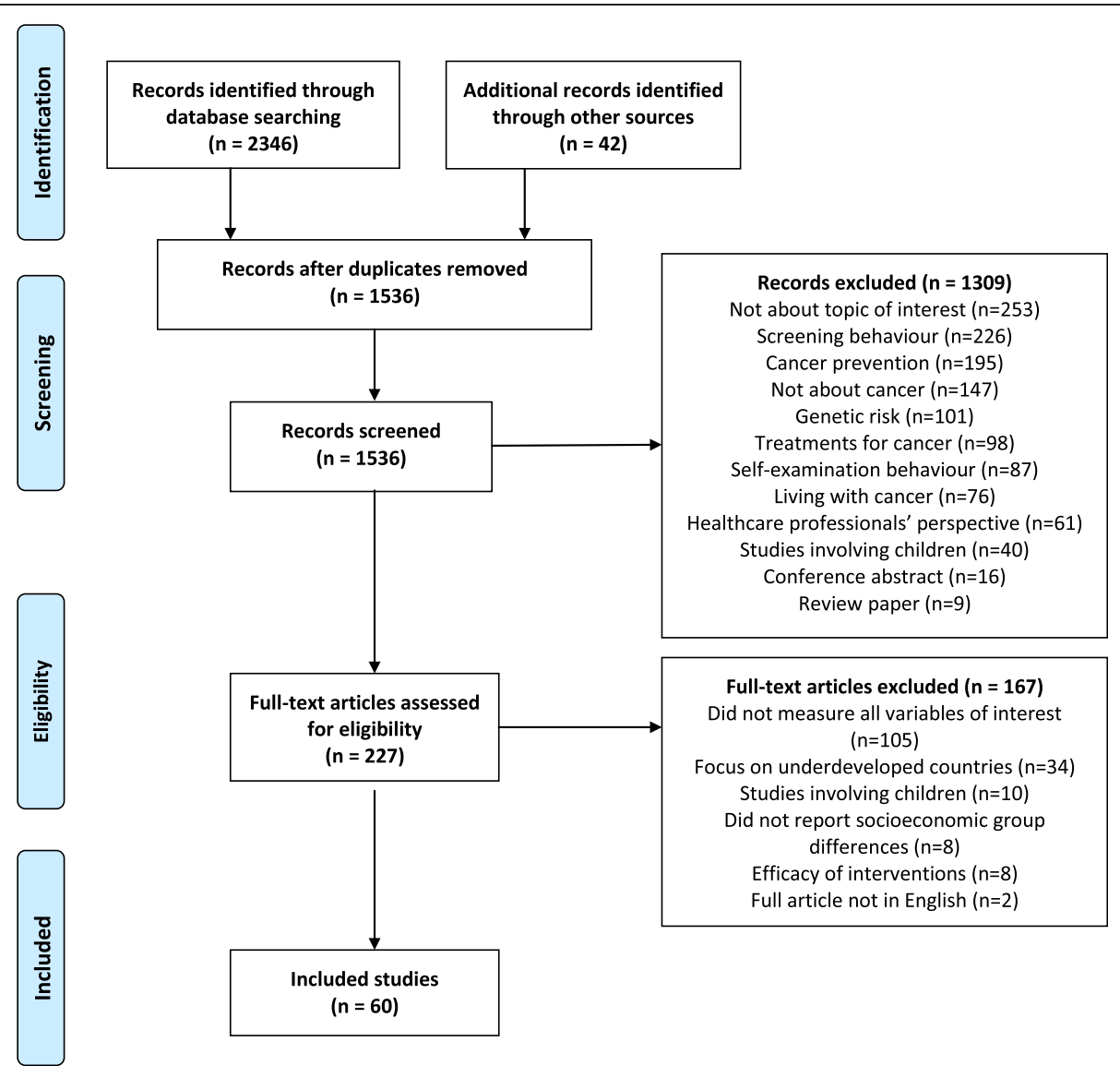

Fig. 2 PRISMA flow diagram. Produced using a downloadable template available at http://www.prisma-statement.org/ (Moher et al, 2009 [22])

agreement between raters (GM and RR) for inclusion/ exclusion of studies, critical appraisal and data extraction was $87 \%$.

A total of 53 studies examined time to symptom presentation, seven studies reported presentation behaviour (if participants did or did not present or anticipate presenting to their doctor with reported symptoms), 45 studies measured actual symptom presentation, 15 studied anticipated symptom presentation, 46 studies assessed knowledge for cancer symptoms, 32 studies explored beliefs about cancer and 50 studies examined perceived barriers/facilitators to symptom presentation. The numbers of studies by tumour site were as follows: breast $(n=22)$, any cancer/multiple tumour sites $(n=15)$, colorectal $(n=7)$, skin $(n=6)$, oral and pharyngeal $(n=3)$, ovarian $(n=3)$, lung $(n=2)$, gynaecological $(n=1)$, and prostate $(n=1)$ (see Table 1$)$. Results are presented according to domain headings.

\section{Symptom presentation}

Studies involving anticipated symptom presentation reported shorter time to symptom presentation compared with studies that examined actual time to symptom presentation. In the former, most participants anticipated seeking medical help within one week [28-30] or within one month [19,31,32], in contrast to real-world studies where it was more common for patients to have waited over two months before seeking medical help [33-41]. The most prompt actual and anticipated symptom presentation was reported for lumps [32, 38, 42-47] or bleeding [19, 32, 48-53]. Studies examining participants who reported experiencing a potential symptom of cancer in the past three months found between $59 \%$ and $75 \%$ of participants had consulted a doctor about their symptom $[49,54,55]$.

Disparity between actual and anticipated symptom presentation relating to socioeconomic group was observed. In five studies, shorter anticipated time to symptom presentation was observed in lower compared to higher socioeconomic groups [19, 28, 31, 32, 48]. Conversely, in two studies, longer anticipated time to symptom presentation was reported in those from lower socioeconomic groups compared with higher socioeconomic groups $[56,57]$. 
Table 1 Table of included studies

\begin{tabular}{|c|c|c|c|c|c|c|c|c|}
\hline Study & Method & Sample & Country & $\begin{array}{l}\text { Tumour } \\
\text { site }\end{array}$ & $\begin{array}{l}\text { Socio- } \\
\text { economic } \\
\text { measure }\end{array}$ & $\begin{array}{l}\text { Measures: Knowledge (K), } \\
\text { Beliefs (B), Perceived barriers } \\
\text { (PB), Perceived facilitators } \\
\text { (PF), Symptomatic } \\
\text { Presentation (SP) }\end{array}$ & $\begin{array}{l}\text { Measure of association } \\
\text { between variables of interest } \\
\text { and socioeconomic indicator } \\
\text { (qualitative studies not } \\
\text { applicable) }\end{array}$ & Quality appraisal \\
\hline Brain et al. (2014) [28] & $\begin{array}{l}\text { Hypothetical } \\
\text { Quantitative }\end{array}$ & $\begin{array}{l}1043 \text { women. } \\
\text { Aged } 50 \text { years } \\
\text { and over }\end{array}$ & Wales & Ovarian & $\begin{array}{l}\text { Postcode, } \\
\text { education }\end{array}$ & $\begin{array}{l}\text { K: Recognition (mean, } 6.85 \\
\text { symptoms) } \\
\text { B: Cancer worry } \\
\text { PB: Emotional and practical } \\
\text { barriers } \\
\text { SP: Sought medical help in } \\
\text { under } 3 \text { weeks } \\
(n=898)\end{array}$ & $\begin{array}{l}\text { K: Lower education } \\
\text { associated with lower } \\
\text { knowledge }(F(2,1005)=8.23, \\
p<0.001) \text {; higher deprivation } \\
\text { (postcode) associated with } \\
\text { lower knowledge }\left(F_{3,886}=2.82 \text {, }\right. \\
p<0.05) \\
\text { B: NR } \\
\text { PB: NR } \\
\text { SP: Higher education } \\
\text { associated with longer time to } \\
\text { SP, }(\mathrm{OR}=2.64, p \leq 0.001) \text {; NS } \\
\text { difference between } \\
\text { deprivation by postcode and } \\
\text { anticipated delay }\left(\mathrm{X}_{(3)}^{2}=6.73,\right. \\
p>0.05) \text { NS }\end{array}$ & Good \\
\hline Brouha et al. (2005) [76] & $\begin{array}{l}\text { Retrospective } \\
\text { Quantitative }\end{array}$ & $\begin{array}{l}189 \text { men and } \\
\text { women. Mean } \\
\text { age: } 59 \text { years }\end{array}$ & Holland & $\begin{array}{l}\text { Oral and } \\
\text { Pharyngeal }\end{array}$ & $\begin{array}{l}\text { Education, } \\
\text { income }\end{array}$ & $\begin{array}{l}\text { K: Symptom interpretation } \\
\text { ('cancer', } n=2 \text { ), misattribution } \\
\text { of symptoms to dental } \\
\text { problems delayed SP } \\
\text { PB: Symptom did not } \\
\text { interfere with daily life } \\
\text { PF: Persistence of symptom, } \\
\text { development of new } \\
\text { symptom } \\
\text { SP: Mean time to symptom } \\
\text { presentation (pharyngeal, } \\
45 \text { days; oral, } 28 \text { days) }\end{array}$ & $\begin{array}{l}\text { K: NR } \\
\text { PB: NR } \\
\text { PF: NR } \\
\text { SP: Education and income } \\
\text { not associated with time to } \\
\text { SP (statistics NR) }\end{array}$ & Medium \\
\hline Burgess et al. (1998) [45] & $\begin{array}{l}\text { Retrospective } \\
\text { Qualitative }\end{array}$ & $\begin{array}{l}185 \text { women. Mean } \\
\text { age: } 54 \text { years }\end{array}$ & UK & Breast & Occupation & $\begin{array}{l}\text { K: Symptom interpretation } \\
\text { ( } 46 \% \text { thought their symptom } \\
\text { indicated cancer) } \\
\text { B: Fear } \\
\text { PF: Symptom disclosure, } \\
\text { appearance of new } \\
\text { symptoms, appointment } \\
\text { booked with GP for another } \\
\text { reason } \\
\text { SP: Waited over } 3 \text { months to } \\
\text { seek medical help ( } 19 \%)\end{array}$ & & Medium \\
\hline Burgess et al. (2000) [67] & $\begin{array}{l}\text { Retrospective } \\
\text { Qualitative }\end{array}$ & $\begin{array}{l}158 \text { women. Mean } \\
\text { age: } 53 \text { years }\end{array}$ & UK & Breast & Occupation & $\begin{array}{l}\text { PB: Life events } \\
\text { SP: Waited over } 3 \text { months to } \\
\text { seek medical help }(18 \%)\end{array}$ & & Medium \\
\hline
\end{tabular}


Table 1 Table of included studies (Continued)

\begin{tabular}{|c|c|c|c|c|c|c|c|c|}
\hline Burgess et al. (2001) [43] & $\begin{array}{l}\text { Retrospective } \\
\text { Qualitative }\end{array}$ & $\begin{array}{l}46 \text { women. Mean } \\
\text { age: } 54.1 \text { years }\end{array}$ & UK & Breast & Occupation & $\begin{array}{l}\text { K: Symptom interpretation } \\
\text { ('lump' most attributed to } \\
\text { cancer) } \\
\text { B: Consequences of } \\
\text { treatment } \\
\text { PB: Not wanting to bother } \\
\text { the doctor, poor health } \\
\text { service utilisation, competing } \\
\text { life priorities } \\
\text { PF: Symptom disclosure, } \\
\text { change in symptom } \\
\text { SP: Waited over } 3 \text { months to } \\
\text { seek medical help }(n=31)\end{array}$ & & Medium \\
\hline $\begin{array}{l}\text { Cameron and Hinton } \\
\text { (1968) [58] }\end{array}$ & $\begin{array}{l}\text { Retrospective } \\
\text { Quantitative }\end{array}$ & 83 women & UK & Breast & $\begin{array}{l}\text { Education, } \\
\text { husband's } \\
\text { occupation }\end{array}$ & $\begin{array}{l}\text { K: Symptom interpretation } \\
\text { B: Fear, worry } \\
\text { SP: } 61 \% \text { sought medical help } \\
\text { within } 1 \text { month }\end{array}$ & $\begin{array}{l}\text { K: NR } \\
\text { B: NR } \\
\text { SP: Higher education } \\
\text { associated with shortest time } \\
\text { to SP for lump symptoms } \\
\left(x^{2}=6.6, p<0.05\right) \text {; Higher } \\
\text { social group (husband's } \\
\text { occupation) associated with } \\
\text { shortest time to SP }\left(x^{2}=3.02 \text {, }\right. \\
p<0.01)\end{array}$ & Poor \\
\hline Caplan (1995) [44] & $\begin{array}{l}\text { Retrospective } \\
\text { Quantitative }\end{array}$ & 162 women & US & Breast & $\begin{array}{l}\text { Income, } \\
\text { education, } \\
\text { employment }\end{array}$ & $\begin{array}{l}\text { PB: Fluctuating symptoms, } \\
\text { relationship with GP } \\
\text { SP: Waited over } 2 \text { months to } \\
\text { seek medical help }(n=27)\end{array}$ & $\begin{array}{l}\text { PB: NR } \\
\text { SP: Lower socioeconomic } \\
\text { group (various indices) } \\
\text { associated with longer time } \\
\text { to SP, but NS: High vs low } \\
\text { income (OR 2.56, } 95 \% \text { Cl: } \\
\text { 0.68-8.64*); High vs low } \\
\text { education (OR 1.07, } 95 \% \text { Cl: } \\
\left.0.41-2.77^{*}\right) \text {; Working vs non- } \\
\text { working (OR 0.72, } 95 \% \text { Cl: } \\
\left.0.27-1.99^{*}\right)\end{array}$ & Poor \\
\hline Carter-Harris et al. (2015) [69] & $\begin{array}{l}\text { Retrospective } \\
\text { Qualitative }\end{array}$ & $\begin{array}{l}11 \text { men }(n=4) \\
\text { and women } \\
(n=7) \text {. Age range: } \\
40-76 \text { years }\end{array}$ & US & Lung & $\begin{array}{l}\text { Education, } \\
\text { employment }\end{array}$ & $\begin{array}{l}\text { K: Symptom interpretations } \\
\text { (one participant was alarmed } \\
\text { at symptoms) } \\
\text { PB: Vague and intermittent } \\
\text { nature of symptoms } \\
\text { PF: Worsening of symptoms, } \\
\text { good relationship with GP } \\
\text { SP: Immediate }(n=1)\end{array}$ & & Medium \\
\hline $\begin{array}{l}\text { Chonjnacka-Szawlowska } \\
\text { et al. (2013) [36] }\end{array}$ & $\begin{array}{l}\text { Retrospective } \\
\text { Quantitative }\end{array}$ & $\begin{array}{l}301 \text { men }(n=186) \\
\text { and women } \\
(n=115) \text {. Mean } \\
\text { age: } 42.3 \text { years }\end{array}$ & Poland & All & Education & $\begin{array}{l}\text { K: Recall, mean: } 1.51 \\
\text { B: Fatalism and cancer } \\
\text { curability } \\
\text { SP: Mean time to symptom } \\
\text { presentation: } 6 \text { months and } \\
10 \text { days; stage of cancer }\end{array}$ & $\begin{array}{l}\text { K: NR } \\
\text { B: NR } \\
\text { SP: NS correlation between } \\
\text { education and stage of } \\
\text { cancer (statistics NR) }\end{array}$ & Medium \\
\hline
\end{tabular}


Table 1 Table of included studies (Continued)

\begin{tabular}{|c|c|c|c|c|c|c|c|c|}
\hline Coates et al. (1992) [42] & $\begin{array}{l}\text { Retrospective } \\
\text { Quantitative }\end{array}$ & $\begin{array}{l}735 \text { women } \\
\text { (410 black and } \\
325 \text { white). Age } \\
\text { range: } 20 \text { to } 79\end{array}$ & US & Breast & $\begin{array}{l}\text { Education, } \\
\text { occupation, } \\
\text { poverty index } \\
\text { (income/no of } \\
\text { people in } \\
\text { household) }\end{array}$ & $\begin{array}{l}\text { K: Symptom interpretation } \\
\text { B: Fatalism } \\
\text { PB: Symptom disclosure, } \\
\text { other comorbid conditions, } \\
\text { appointment with doctor } \\
\text { booked for another reason } \\
\text { SP: Median time to symptom } \\
\text { presentation (black women, } \\
16 \text { days; white women, } \\
14 \text { days) }\end{array}$ & $\begin{array}{l}\text { K: NR } \\
\text { B: NR } \\
\text { PB: NR } \\
\text { SP: Higher education } \\
\text { associated with shorter time } \\
\text { to SP (Mantel-cox 1.43, } 95 \% \\
\text { Cl: } 1.11-1.86, p<0.05) \text {, Low } \\
\text { deprivation (poverty index) } \\
\text { associated with shorter time } \\
\text { to SP (Mantel-Cox 1.24, } 95 \% \\
\text { Cl: } 1-1.54, p<0.05 \text { ) }\end{array}$ & Good \\
\hline Cockburn et al. (2003) [54] & $\begin{array}{l}\text { Retrospective } \\
\text { Quantitative }\end{array}$ & $\begin{array}{l}1332 \text { men }(40 \%) \\
\text { and women }(60 \%) \text {. } \\
\text { Aged } 40 \text { years } \\
\text { and over }\end{array}$ & Australia & $\begin{array}{l}\text { Colorectal } \\
\text { (Bowel) }\end{array}$ & Education & $\begin{array}{l}\text { K: Recall ( } 25 \% \text { could } \\
\text { not recall any symptoms), } \\
\text { symptom interpretation } \\
\text { B: Benefits of early diagnosis } \\
\text { SP: } 306 \text { had experienced a } \\
\text { symptom, } 31.9 \% \text { did not } \\
\text { seek medical help }\end{array}$ & $\begin{array}{l}\text { K: Higher education } \\
\text { associated with higher K of } \\
\text { symptoms (PR 0.93, } 95 \% \text { Cl: } \\
0.89-0.96^{*} \text { ) } \\
\text { B: Higher education more } \\
\text { likely to hold positive beliefs } \\
\text { about the benefits of early } \\
\text { diagnosis (statistics NR) } \\
\text { SP: NR }\end{array}$ & Medium \\
\hline Esteva et al. (2013) [70] & $\begin{array}{l}\text { Retrospective } \\
\text { Quantitative }\end{array}$ & $\begin{array}{l}795 \text { men }(n=489) \\
\text { and women } \\
(n=291)\end{array}$ & Spain & Colorectal & $\begin{array}{l}\text { Social class, } \\
\text { education }\end{array}$ & $\begin{array}{l}\text { K: Symptom interpretation } \\
\text { ('not serious', } 65.6 \% \text { ) } \\
\text { PF: Symptom disclosure, } \\
\text { good relationship with } \\
\text { GP (trust) } \\
\text { SP: Median time to symptom } \\
\text { presentation (19 days) }\end{array}$ & $\begin{array}{l}\text { K: NR } \\
\text { SP: NS association between } \\
\text { social class and time to SP } \\
\text { (statistics NR), NS association } \\
\text { between education and time } \\
\text { to SP (statistics NR) }\end{array}$ & Medium \\
\hline $\begin{array}{l}\text { Facione and Facione } \\
\text { (2006) [59] }\end{array}$ & $\begin{array}{l}\text { Retrospective } \\
\text { Qualitative }\end{array}$ & $\begin{array}{l}28 \text { women. Mean } \\
\text { age: } 42.34 \text { years }\end{array}$ & US & Breast & $\begin{array}{l}\text { Income, } \\
\text { education, } \\
\text { health } \\
\text { insurance }\end{array}$ & $\begin{array}{l}\text { K: Symptom interpretation } \\
\text { B: Fear, fatalism, benefits of } \\
\text { early diagnosis } \\
\text { PB: Worry about losing } \\
\text { relationship with partner if } \\
\text { diagnosed with cancer } \\
\text { PF: Symptom disclosure } \\
\text { SP: Sought medical help after } \\
3 \text { months }(n=15)\end{array}$ & & Medium \\
\hline Facione et al. (2002) [56] & $\begin{array}{l}\text { Hypothetical } \\
\text { Quantitative }\end{array}$ & $\begin{array}{l}669 \text { women. Mean } \\
\text { age: } 46.95 \text { years }\end{array}$ & US & Breast & $\begin{array}{l}\text { Income, } \\
\text { education, } \\
\text { health care } \\
\text { insurance }\end{array}$ & $\begin{array}{l}\text { K: Recognition ( } 10 \% \\
\text { recognised all or all but one } \\
\text { symptoms) } \\
\text { B: Fatalism } \\
\text { PB: Difficulties with access, } \\
\text { prejudice in health care, } \\
\text { concerns about deportation, } \\
\text { use of alternative therapies } \\
\text { SP: Likely to delay ( } 23.7 \%) \text {. }\end{array}$ & $\begin{array}{l}\text { K: Higher education } \\
\text { associated with higher } \\
\text { symptom recognition } \\
\left(F_{3,690}=32.32, p<0.001\right) \\
\text { B: NR } \\
\text { PB: NR } \\
\text { SP: Lack of insurance } \\
\text { associated with longer time } \\
\text { to SP (Cramer's } V=0.187 \text {, } \\
p<0.001) \text {; Lower education } \\
\text { associated with longer time } \\
\text { to SP (Cramer's } V=0.288 \text {, } \\
p<0.001) \text {; Lower income }\end{array}$ & Good \\
\hline
\end{tabular}


Table 1 Table of included studies (Continued)

\begin{tabular}{|c|c|c|c|c|c|c|c|c|}
\hline & & & & & & & $\begin{array}{l}\text { associated with longer time } \\
\text { to } S P(C r a m e r ' s ~ V=0.291 \text {, } \\
p<0.001)\end{array}$ & \\
\hline Facione et al. (1997) [84] & $\begin{array}{l}\text { Hypothetical } \\
\text { Quantitative }\end{array}$ & $\begin{array}{l}352 \text { African } \\
\text { American or Black } \\
\text { women. Mean } \\
\text { age: } 38.6 \text { years }\end{array}$ & US & Breast & $\begin{array}{l}\text { Income, } \\
\text { Education, } \\
\text { Employment }\end{array}$ & $\begin{array}{l}\text { B: Fear, fatalism } \\
\text { PB: Poor health service } \\
\text { utilization } \\
\text { SP: } 11.6 \%=\text { strong } \\
\text { disposition to SP. }\end{array}$ & $\begin{array}{l}\text { B: NR } \\
\text { PB: NR } \\
\text { SP: Stronger disposition to SP } \\
\text { associated with lower } \\
\text { education }(r=0.19, p<0.01) \\
\text { and lower income }(r=0.32 \\
p<0.001)\end{array}$ & Medium \\
\hline Fitzpatrick et al. (1998) [57] & $\begin{array}{l}\text { Hypothetical } \\
\text { Quantitative }\end{array}$ & $\begin{array}{l}280 \text { men. Mean } \\
\text { age: } 53.7 \text { years }\end{array}$ & Ireland & Prostate & $\begin{array}{l}\text { Health } \\
\text { insurance, } \\
\text { occupation }\end{array}$ & $\begin{array}{l}\text { B: Fear } \\
\text { PB: Poor health service } \\
\text { utilisation, dislike of doctors, } \\
\text { embarrassment } \\
\text { SP: } 81 \% \text { would seek medical } \\
\text { help if developed urinary } \\
\text { symptoms }\end{array}$ & $\begin{array}{l}\text { B: NR } \\
\text { PB: NR } \\
\text { SP: Non-manual social class } \\
\text { associated with higher } \\
\text { willingness to attend GP with } \\
\text { symptoms (OR } 1.8, p<0.05^{* *} \text { ) }\end{array}$ & Good \\
\hline Forbes et al. (2011) [29] & $\begin{array}{l}\text { Hypothetical } \\
\text { Quantitative }\end{array}$ & $\begin{array}{l}1515 \text { women from } \\
\text { various ethnic } \\
\text { groups (White, } \\
\text { South Asian, } \\
\text { Black). Aged } 30 \\
\text { years and over }\end{array}$ & UK & Breast & $\begin{array}{l}\text { Postcode } \\
\text { (IMD) }\end{array}$ & $\begin{array}{l}\text { K: Recognition ( } 18 \% \\
\text { recognised } 5 \text { or more } \\
\text { non-lump symptoms) } \\
\text { PB: self-efficacy, worry what } \\
\text { the doctor might find, } \\
\text { embarrassment, worry } \\
\text { about wasting doctors } \\
\text { time, difficulty getting an } \\
\text { appointment } \\
\text { SP: } 73 \% \text { would seek help } \\
\text { within } 1 \text { week }\end{array}$ & $\begin{array}{l}\text { K: Differences between ethnic } \\
\text { groups for cancer awareness } \\
\text { not due to IMD score or } \\
\text { lower level of education } \\
\text { (statistics NR) } \\
\text { PB: Differences between } \\
\text { ethnic groups for PB not due } \\
\text { to IMD score (statistics NR) } \\
\text { SP: NR }\end{array}$ & Good \\
\hline Forbes et al. (2014) [64] & $\begin{array}{l}\text { Retrospective } \\
\text { Quantitative }\end{array}$ & $\begin{array}{l}1999 \text { men ( } n= \\
1077) \text { and women } \\
(n=922) . \text { Aged } 50 \\
\text { or over }\end{array}$ & UK & All & Postcode & $\begin{array}{l}\text { K: Symptom interpretation } \\
\text { PB: } 48 \% \text { of patients reported } \\
\text { at least one barrier } \\
\text { SP: Delay over } 3 \text { months } \\
(n=21 \%)\end{array}$ & $\begin{array}{l}\text { B: NR } \\
\text { PB: NR } \\
\text { SP: Lowest socioeconomic } \\
\text { group associated with } \\
\text { longest time to SP }(1.51 \text {, } \\
95 \% \text { Cl: 1.18-1.88*) }\end{array}$ & Good \\
\hline
\end{tabular}


Table 1 Table of included studies (Continued)

\begin{tabular}{|c|c|c|c|c|c|c|c|c|}
\hline & & & & & & $\begin{array}{l}\text { SP: Mean time to symptom } \\
\text { presentation ( } 9 \text { months) }\end{array}$ & $\begin{array}{l}\text { to SP (Fishers Exact test, } \\
p<0.01^{* *} \text { ) }\end{array}$ & \\
\hline Goldsen et al. (1957) [61] & $\begin{array}{l}\text { Retrospective } \\
\text { Quantitative }\end{array}$ & $\begin{array}{l}727 \text { men and } \\
\text { women }\end{array}$ & US & All & $\begin{array}{l}\text { Income, } \\
\text { education and } \\
\text { occupation }\end{array}$ & $\begin{array}{l}\text { K: Symptom interpretation } \\
\text { ( } 20 \% \text { thought symptoms } \\
\text { indicated cancer) } \\
\text { B: Cancer worry, fatalism } \\
\text { PB: Poor health service } \\
\text { utilization, symptom not } \\
\text { noticed } \\
\text { PF: Symptom disclosure } \\
\text { SP: } 51.3 \% \text { sought medical } \\
\text { help under } 30 \text { days }\end{array}$ & $\begin{array}{l}\text { K: NR } \\
\text { B: NR } \\
\text { PB: NR } \\
\text { PF: NR } \\
\text { SP: Lower income, education } \\
\text { and occupation associated } \\
\text { with longest time to SP } \\
\text { (statistics NR) }\end{array}$ & Medium \\
\hline Gould et al. (2010) [39] & $\begin{array}{l}\text { Retrospective } \\
\text { Qualitative }\end{array}$ & $\begin{array}{l}14 \text { women. Aged } \\
\text { range: } 30 \text { to } 69 \\
\text { years }\end{array}$ & Canada & Breast & $\begin{array}{l}\text { Education, } \\
\text { employment, } \\
\text { income }\end{array}$ & $\begin{array}{l}\text { K: Symptom interpretation } \\
\text { (poor for non-lump } \\
\text { symptoms) } \\
\text { B: Fear } \\
\text { PB: Previous benign disease, } \\
\text { watchful waiting, competing } \\
\text { life priorities } \\
\text { PF: Symptom disclosure, } \\
\text { already have another } \\
\text { appointment booked. } \\
\text { SP: All women waited } \\
\text { 8+ weeks }\end{array}$ & & Medium \\
\hline Grant et al. (2010) [82] & $\begin{array}{l}\text { Retrospective } \\
\text { Qualitative }\end{array}$ & $\begin{array}{l}15 \text { men }(n=7) \\
\text { and women } \\
(n=8) . \text { Aged } 45 \\
\text { years and under }\end{array}$ & Scotland & Oral & Postcode & $\begin{array}{l}\text { K: Symptom interpretation } \\
\text { PB: Self-medication } \\
\text { PF: Already had an } \\
\text { appointment booked } \\
\text { SP: Sought medical help } \\
\text { within } 8 \text { weeks }(n=8)\end{array}$ & & Medium \\
\hline Greer (1974) [68] & $\begin{array}{l}\text { Retrospective } \\
\text { Quantitative }\end{array}$ & $\begin{array}{l}160 \text { women with } \\
\text { stage I or stage II } \\
\text { cancer. Aged } 70 \\
\text { years and under }\end{array}$ & UK & Breast & Social Class & $\begin{array}{l}\text { K: Symptom interpretation } \\
\text { B: Fear, fatalism } \\
\text { PB: Embarrassment } \\
\text { SP: } 64 \% \text { sought medical } \\
\text { help within } 1 \text { month }\end{array}$ & $\begin{array}{l}\text { K: NR } \\
\text { B: NR } \\
\text { PB: NR } \\
\text { SP: NS difference between } \\
\text { time to SP and social class } \\
\text { (statistics NR) }\end{array}$ & Poor \\
\hline Hunter et al. (2003) [30] & $\begin{array}{l}\text { Hypothetical } \\
\text { Quantitative }\end{array}$ & $\begin{array}{l}546 \text { women. Mean } \\
\text { age: } 47 \text { years }\end{array}$ & UK & Breast & Occupation & $\begin{array}{l}\text { K: Recognition (good, } \\
\text { mean 6.65)B: Beliefs about } \\
\text { treatment } \\
\text { SP: } 58.6 \% \text { would seek } \\
\text { immediate medical help. }\end{array}$ & $\begin{array}{l}\text { K: NR } \\
\text { NR: NR } \\
\text { SP: Socioeconomic group not } \\
\text { associated with time to SP } \\
\left(F_{(1,518)}=0.29, p>0.05\right)\end{array}$ & Medium \\
\hline Kakagia et al. (2013) [34] & $\begin{array}{l}\text { Retrospective } \\
\text { Quantitative }\end{array}$ & $\begin{array}{l}513 \text { men } \\
(n=56.5 \%) \text { and } \\
\text { women } \\
(n=43.5 \%) . \\
\text { Mean age: } 67.5 \\
\text { years }\end{array}$ & Greece & Skin & $\begin{array}{l}\text { Education, } \\
\text { ethnicity, area } \\
\text { of residence }\end{array}$ & $\begin{array}{l}\text { K: Symptom interpretation } \\
\text { B: Fear, fatalism } \\
\text { PB: Other serious } \\
\text { comorbidities, poor health } \\
\text { service utilisation, dislike of } \\
\text { doctors and hospitals, } \\
\text { transport issues, worry about }\end{array}$ & $\begin{array}{l}\text { K: NR } \\
\text { B: NR } \\
\text { PB: NR } \\
\text { PF: NR } \\
\text { SP: Longer time to SP } \\
\text { associated with lower } \\
\text { socioeconomic group }\end{array}$ & Medium \\
\hline
\end{tabular}


Table 1 Table of included studies (Continued)

wasting doctors time,

embarrassment, competing

life demands

PF: Symptom disclosure,

active encouragement to

seek medical help

SP: Mean time to symptom presentation (3.9 months)

Lam et al. (2009) [63]

Retrospective Qualitative

37 women. Age range 20-81 years

Hong Kong

Breast

Employment education

k: Symptom interpretation B: fear, fatalism

PB: Watchful waiting, poor general health service

utilisation, cost, competing

life priorities, embarrassment

PF: Persistence of symptoms,

appearance of new

symptom, symptom

disclosure, symptom

interfering with daily life,

appointment booked for

another reason

SP: Waited over 3 months to

seek medical help $(n=14)$ age: 51.97 years

(OR 1.89, 95 \% Cl: 0.9-3.8.

$p<0.001)$ and lower

education (OR 3.01, $95 \% \mathrm{Cl}$.

1.6-5.6, $p<0.001$

128 men $(n=33) \quad$ US

and women

$(n=95)$. Mean

age: 63 years

Low et al. (2013) [31]

Hypothetical

1000 women

Mean age:

UK

47 years
Employment,

education

B: Fear

B: Cost, gender of doctor,

unsure where to seek

medical help, competing life

priorities, no history of breast

problems, symptom

disclosure

PF: Symptom disclosure

SP: Median time to sy

presentation (14 days)

B: NR

B: Symptom disclosure for

women with lower education

ss likely to translate into

mmediate SP $\left(x^{2}=6.4\right.$

d.f. $=2, p<0.05$ )

SP: Longer time to SP

associated with higher

education (OR 3.35, 95 \%

Cl:1.19-9.42, $p<0.05)$ and full

time employment (OR 2.52,

$95 \%$ Cl: 1.18-5.36, $p<0.05$ )

Employment, B: Curability of cancer, cancer

income, is contagious, surgery causes

education cancer to spread

SP: Poor for non-specific

symptoms

Education, car K: Recall (poor, mean 0.6) and K: NR

ownership, recognition (good, mean 6.3) PB: NR

home- PB. Mean number of barriers

B: Mean number of barriers

$\begin{array}{ll}\text { endorsed (2.2), emotional, } & \text { group associated with longe } \\ \text { practical and service barriers } & \text { time to SP (beta }=0.12, \text { SE }\end{array}$

SP. Higher socioeconomic SP: Varied by symptom, most $\left.0.05, p<0.001^{* *}\right)$

Medium

Good

Medium 
Table 1 Table of included studies (Continued)

\begin{tabular}{|c|c|c|c|c|c|c|c|c|}
\hline & & & & & & $\begin{array}{l}\text { would seek help under } 2 \\
\text { weeks }\end{array}$ & & \\
\hline Magarey et al. (1977) [72] & $\begin{array}{l}\text { Retrospective } \\
\text { Quantitative }\end{array}$ & $\begin{array}{l}64 \text { women. Age in } \\
\text { years: less than } 40 \\
(n=13), 40-60 \\
(n=28), \text { over } 60 \\
(n=23) .\end{array}$ & Australia & Breast & Education & $\begin{array}{l}\text { PB: Denial, anxiety } \\
\text { SP: Most sought medical help } \\
\text { within } 2 \text { weeks }(n=35)\end{array}$ & $\begin{array}{l}\text { PB: NR } \\
\text { SP: Education not associated } \\
\text { with time to SP (statistics NR) }\end{array}$ & Poor \\
\hline Marlow et al. (2014) [78] & $\begin{array}{l}\text { Hypothetical } \\
\text { Qualitative }\end{array}$ & $\begin{array}{l}54 \text { women from } \\
\text { ethnic minority } \\
\text { groups living with } \\
\text { a comparison of } \\
\text { white women. } \\
\text { Age range: } 25-64 \\
\text { years }\end{array}$ & UK & $\begin{array}{l}\text { Breast and } \\
\text { Ovarian }\end{array}$ & $\begin{array}{l}\text { Employment, } \\
\text { education, } \\
\text { living } \\
\text { arrangement }\end{array}$ & $\begin{array}{l}\text { K: Recall (good for lumps/ } \\
\text { bleeding, poor for other } \\
\text { symptoms) } \\
\text { B: Fear, fatalism, benefits of } \\
\text { early diagnosis } \\
\text { PB: Poor relationship with GP, } \\
\text { emotional barriers, practical } \\
\text { barriers, service barriers, } \\
\text { competing life priorities } \\
\text { PF: Symptom disclosure } \\
\text { SP: Varied: days to months. } \\
\text { All sought help within } \\
3 \text { months. }\end{array}$ & & Medium \\
\hline McCaffery et al. (2003) [50] & $\begin{array}{l}\text { Hypothetical } \\
\text { Quantitative }\end{array}$ & $\begin{array}{l}1637 \text { men } \\
(n=763) \text { and } \\
\text { women }(n=874) . \\
\text { Age range: 16-74 } \\
\text { years }\end{array}$ & UK & Colorectal & Education & $\begin{array}{l}\text { K: Recall (poor) } \\
\text { B: Fear } \\
\text { SP: } 92.8 \% \text { would anticipate } \\
\text { seeking medical help if } \\
\text { noticed blood in stool for } \\
\text { more than } 2 \text { weeks. }\end{array}$ & $\begin{array}{l}\text { K: Higher education } \\
\text { associated with higher } \\
\text { symptom recall }\left(x^{2}[4]=73.98 \text {, }\right. \\
p<0.001) \\
\text { B: Lower education } \\
\text { associated with most } \\
\text { negative beliefs ( } x^{2}[4]= \\
74.96, p<0.001 \text { ) } \\
\text { SP: NS association with } \\
\text { education and SP intentions } \\
\text { (statistics NR) }\end{array}$ & Good \\
\hline Meechan et al. (2003) [46] & $\begin{array}{l}\text { Retrospective } \\
\text { Mixed }\end{array}$ & $\begin{array}{l}85 \text { women. Mean } \\
\text { age: } 38.9 \text { years }\end{array}$ & New Zealand & Breast & Education & $\begin{array}{l}\text { PB: Having a family member } \\
\text { with cancer, low emotional } \\
\text { response to symptom } \\
\text { PF: High emotional response } \\
\text { to symptom } \\
\text { SP: Median time to symptom } \\
\text { presentation } \\
\text { (14 days) }\end{array}$ & $\begin{array}{l}\text { PB: NR } \\
\text { PF: NR } \\
\text { SP: NS association between } \\
\text { education and time to SP ( } \\
(83)=-1.26, p>0.05)\end{array}$ & Medium \\
\hline Mor (1990) [74] & $\begin{array}{l}\text { Retrospective } \\
\text { Mixed }\end{array}$ & $\begin{array}{l}700 \text { patients. Age } \\
\text { range: } 45 \text { to } 90 \\
\text { years }\end{array}$ & US & $\begin{array}{l}\text { Lung, } \\
\text { Breast and } \\
\text { Colorectal }\end{array}$ & $\begin{array}{l}\text { Education, } \\
\text { housing, } \\
\text { income, } \\
\text { education }\end{array}$ & $\begin{array}{l}\text { K: Symptom interpretation } \\
\text { (best knowledge for breast } \\
\text { cancer patients) } \\
\text { B: Fear ( } 16.8 \% \text { of delayers) } \\
\text { PB: "thought it would go } \\
\text { away" (60.5 \% of delayers), } \\
\text { too busy ( } 8.4 \% \text { of delayers) }\end{array}$ & $\begin{array}{l}\text { K: NR } \\
\text { B: NR } \\
\text { PB: NR } \\
\text { SP: NS relationship between } \\
\text { socioeconomic group and } \\
\text { time to SP (statistics NR) }\end{array}$ & Medium \\
\hline
\end{tabular}


Table 1 Table of included studies (Continued)

\begin{tabular}{|c|c|c|c|c|c|c|}
\hline & & & & & & colorectal (87.6\%) \\
\hline Oliveria et al. (1999) [37] & $\begin{array}{l}\text { Retrospective } \\
\text { Quantitative }\end{array}$ & $\begin{array}{l}255 \text { men and } \\
\text { women. Aged } 18 \\
\text { years and over }\end{array}$ & US & Melanoma & $\begin{array}{l}\text { Education, } \\
\text { insurance }\end{array}$ & $\begin{array}{l}\text { K: Recognition (poor) } \\
\text { SP: Mean time to symptom } \\
\text { presentation ( } 2 \text { months) }\end{array}$ \\
\hline $\begin{array}{l}\text { O'Mahony and Hegarty } \\
\text { (2009) [47] }\end{array}$ & $\begin{array}{l}\text { Retrospective } \\
\text { Quantitative }\end{array}$ & $\begin{array}{l}99 \text { women. Mean } \\
\text { age: } 40 \text { years }\end{array}$ & Ireland & Breast & $\begin{array}{l}\text { Employment, } \\
\text { education }\end{array}$ & $\begin{array}{l}\text { K: Symptom interpretation } \\
\text { PB: Competing life priorities, } \\
\text { emotional reactions to } \\
\text { symptom (afraid, scared, } \\
\text { unsure) } \\
\text { PF: Symptom disclosure, } \\
\text { anxiety } \\
\text { SP: Waited over } 1 \text { month to } \\
\text { seek medical help }(n=26)\end{array}$ \\
\hline O'Mahony et al. (2011) [79] & $\begin{array}{l}\text { Retrospective } \\
\text { Qualitative }\end{array}$ & $\begin{array}{l}10 \text { women. Mean } \\
\text { age: } 40 \text { years }\end{array}$ & Ireland & Breast & $\begin{array}{l}\text { Education, } \\
\text { employment, } \\
\text { insurance }\end{array}$ & $\begin{array}{l}\text { K: Most aware that a lump } \\
\text { was a symptomof cancer } \\
\text { B: Fatalism, curability of } \\
\text { cancer, fear } \\
\text { PB: Denial, competing life } \\
\text { priorities } \\
\text { PF: Symptom disclosure, } \\
\text { good perceived access to GP, } \\
\text { good relationship with GP } \\
\text { SP: Sought medical help } \\
\text { within } 1 \text { month }(n=6)\end{array}$ \\
\hline Pedersen et al. (2011) [85] & $\begin{array}{l}\text { Retrospective } \\
\text { Quantitative }\end{array}$ & $\begin{array}{l}901 \text { men }(n=423) \\
\text { and women } \\
(n=487) . \text { Mean } \\
\text { age: } 61.8 \text { years }\end{array}$ & Denmark & All & Education & $\begin{array}{l}\text { PF: Symptom disclosure, } \\
\text { good partner support } \\
\text { SP: Median interval: } 12 \text { days }\end{array}$ \\
\hline Quaife et al. (2014) [32] & $\begin{array}{l}\text { Hypothetical } \\
\text { Quantitative }\end{array}$ & $\begin{array}{l}6965 \text { men } \\
(n=4330) \text { and } \\
\text { women }(n=265) \text {. } \\
\text { Aged } 50 \text { and over }\end{array}$ & UK & All & Education & $\begin{array}{l}\text { K: Recognition (best for } \\
\text { 'lump') } \\
\text { PB: Poor access health } \\
\text { services } \\
\text { SP: Would wait } 2+\text { weeks: } \\
\text { (cough, } n=48.1 \% \text {; breast } \\
\text { change, } n=8.2 \% \text {; rectal } \\
\text { bleeding, } n=7.4 \% \text { ) }\end{array}$ \\
\hline
\end{tabular}

SP: Waited over 3 months to

seek medical help: lung

$(54.9 \%)$, breast $(56.2 \%)$,

colorectal $(87.6 \%)$

Education not associated with time to SP (statistics NR)

K.NR

PB: NR

SP: Higher education

ssociated with longer time

to SP (statistics NR)

\section{Medium}

Medium

PF: NR

SP: NS association between

education and time to SP:

Lower secondary education

and long SP ( $>55$ days) (RRR

$0.79,95 \%$ Cl: 0.36-1.74

$p>0.05)$; tertiary education and long SP ( $>55$ days) (RRR 1.30, $95 \%$ Cl: 0.55-3.08$$
p>0.05)
$$

k: Lower education

associated with lower

ecognition for all 3

symptoms $\left(x^{2}, p<0.05^{* *}\right)$

PB: NR

SP: Lower education

associated with shorter time

to SP for cough (OR 0.61 ,

$95 \%$ Cl: $0.54-0.68, p<0.001$ )

and breast changes (OR 0.68 ,

$95 \%$ Cl: $0.52-0.89, p<0.001$ ).

NS association with 
Table 1 Table of included studies (Continued)

Table 1 Table of included studies (Continued)

\begin{tabular}{|c|c|c|c|c|c|c|c|c|}
\hline \multirow[b]{2}{*}{ Rauscher et al. (2010) [66] } & \multirow[b]{2}{*}{$\begin{array}{l}\text { Retrospective } \\
\text { Quantitative }\end{array}$} & \multirow[b]{2}{*}{$\begin{array}{l}438 \text { women. Age } \\
\text { range: } 30 \text { to } 79 \\
\text { years }\end{array}$} & \multirow[b]{2}{*}{ US } & \multirow[b]{2}{*}{ Breast } & \multirow[b]{2}{*}{$\begin{array}{l}\text { Education, } \\
\text { household } \\
\text { income, health } \\
\text { insurance } \\
\text { status }\end{array}$} & \multirow[b]{2}{*}{$\begin{array}{l}\text { K: Breast lump } \\
\text { misconceptions ( } 20 \% \\
\text { reported one or more } \\
\text { misconception) } \\
\text { PB: Poor general health } \\
\text { service utilisation } \\
\text { SP: Waited over } 3 \text { months to } \\
\text { seek medical help (16\%) }\end{array}$} & \multicolumn{2}{|l|}{$\%$ Cl: $0.67-1.03, p>0.05)$} \\
\hline & & & & & & & $\begin{array}{l}\text { K: Lower income and } \\
\text { education associated with } \\
\text { more breast lump } \\
\text { misconceptions }\left(x^{2}, p<0.001^{* *}\right) \\
\text { PB: NR } \\
\text { SP: Longer time to SP } \\
\text { associated with lower } \\
\text { education }\left(x^{2}, p<0.05^{* *}\right) \text { and } \\
\text { lower income } \\
\left(x^{2}, p<0.05^{* *}\right)\end{array}$ & Medium \\
\hline Ristvedt et al. (2014) [33] & $\begin{array}{l}\text { Retrospective } \\
\text { Quantitative }\end{array}$ & $\begin{array}{l}112 \text { men }(n=55) \\
\text { and women } \\
(n=57) . \text { Mean } \\
\text { age: } 59.3 \text { years }\end{array}$ & US & Colorectal & $\begin{array}{l}\text { Income, area } \\
\text { of residence, } \\
\text { education, } \\
\text { health } \\
\text { insurance }\end{array}$ & $\begin{array}{l}\text { K: Symptom interpretation } \\
\text { ( } 70.5 \% \text { thought symptom } \\
\text { serious within } 13 \text { weeks } \\
\text { post onset) } \\
\text { SP: Median time to symptom } \\
\text { presentation ( } 10 \text { weeks) }\end{array}$ & $\begin{array}{l}\text { K: NR } \\
\text { SP: NS association between } \\
\text { socioeconomic group } \\
\text { (education and household } \\
\text { income) and time to SP } \\
\text { (statistics NR) }\end{array}$ & Medium \\
\hline $\begin{array}{l}\text { Ristvedt and Trinkhaus } \\
\text { (2005) [9] }\end{array}$ & $\begin{array}{l}\text { Retrospective } \\
\text { Quantitative }\end{array}$ & $\begin{array}{l}69 \text { men }(n=42) \\
\text { and women } \\
(n=27) . \text { Mean } \\
\text { age: } 61.3 \text { years }\end{array}$ & US & Colorectal & Education & $\begin{array}{l}\text { K: Symptom interpretation } \\
\text { ('not cancer', } 71 \% \text { ) } \\
\text { PB: Personality (low trait } \\
\text { anxiety), poor health service } \\
\text { utilisation } \\
\text { SP: Mean time to symptom } \\
\text { presentation ( } 25 \text { weeks) }\end{array}$ & $\begin{array}{l}\text { K: NR } \\
\text { PB: NR } \\
\text { SP: Lower education } \\
\text { associated with longer time } \\
\text { to SP (Kaplan-Meier: median } \\
\left.15 \text { weeks, } 95 \% \text { Cl: } 9.0-26.0^{*}\right) \text {; } \\
\text { higher education associated } \\
\text { with shorter time to SP } \\
\text { (Kaplan-Meier: median } 8 \\
\text { weeks, } 95 \% \text { Cl: } 4.0-15.0^{*} \text { ) }\end{array}$ & Medium \\
\hline
\end{tabular}

education and time to SP for

rectal bleeding (OR 0.83, 95

Cl: $0.67-1.03, p>0.05$

misconceptions $\left(x^{2}, p<0.001^{* *}\right)$

NS

B: Those with higher

B: Fear

life priorities (work and family self-detect melanoma

commitments), melanoma $\quad\left(x^{2}, p<0.01^{* *}\right)$

ot detected by participan

PP: NS association with and

ime to SP and socioeconomic

$\begin{array}{ll}\text { PB: Low anxiety, poor general } & \text { K: NR } \\ \text { health service utilisation } & \text { PB: NR }\end{array}$

PF: Symptom disclosure, SP: NS association between

.

socioeconomic group and

of patients

medical help

K: Symptom interpretation

. $0.5 \%$ thought symptom

health post onset)

education,

Symptom interpretation

('not cancer', 71 \%)

SP. Mean time to symptom

weeks, 95 
Table 1 Table of included studies (Continued)

Robb et al. (2009) [19]

2216 men

men

UK

$(n=968)$ and

All

All

Education,

K: Recall (poor, mean = 2.2)

mean $=7.2$ )

PB: Emotional and service

barriers most endorsed

SP: Most would seek medica

help within 2 weeks

K: Higher socioeconomic

group (occupation)

associated with highest

(

knowledge $(F(2,2015)=$

$20.31, p<0.001)$

PB: Lower socioeconomic

group (occupation)

associated with more

emotional barriers endorsed:

worry what the doctor might

find' $\left(x^{2}(1,1989)=17.08\right.$

$p<0.001)$, 'too embarrassed'

$\left(x^{2}(1,1993)=20.74, p<0.001\right)$

'not confident to talk about

symptom' $\left(x^{2}(1,1992)=4.77\right.$

$p<0.05)$, NS association with

'too scared' $\left(x^{2}(1,1977)=1.82\right.$,

$p>0.05)$; Higher

socioeconomic group

(occupation) associated with

more practical barriers

endorsed: 'too busy'

$\left(x^{2}(1,2005)=59.0, p<0.001\right)$

'other things to worry about'

$\left(x^{2}(1,1996)=15.34, p<0.001\right)$

'difficult to arrange transport'

$\left(x^{2}(1,2010)=11.13, p<0.001\right)$

NS association between

socioeconomic group

(occupation) and service

barriers: 'difficult to make

appointment' $\left(x^{2}(1,1983)=\right.$

$0.41, p>0.05)$, 'worried about

wasting the doctors time' $\left(x^{2}\right.$

$(1,1995)=1.44, p>0.05)$

'difficult to arrange transport'

$\left(x^{2}(1,1938)=1.15, p>0.05\right)$

SP: Lower socioeconomic

group (occupation)

associated with shorter time

to SP for unexplained

bleeding $\left(x^{2}(1,1991)=5.82, p\right.$

$<0.01)$, difficulty swallowing

$\left(x^{2}(1,1987)=28.41, p<0.001\right)$

$\operatorname{lump}\left(x^{2}(1,1988)=21.26\right.$,

$p<0.001)$, change in mole $\left(x^{2}\right.$

$(1,1967)=24.24, p<0.001)$

unexplained pain $\left(x^{2}(1,1965)=\right.$

$20.24, p<0.001)$, sore that

does not heal $\left(x^{2}(1,1977)\right.$

$35.84, p<0.001)$, change in 
Table 1 Table of included studies (Continued)

\begin{tabular}{|c|c|c|c|c|c|c|c|c|}
\hline Samet et al. (1988) [62] & $\begin{array}{l}\text { Retrospective } \\
\text { Quantitative }\end{array}$ & $\begin{array}{l}800 \text { men }(n=396) \\
\text { and women } \\
(n=404) . \text { Mean } \\
\text { age: } 72.2 \text { years }\end{array}$ & US & All & $\begin{array}{l}\text { Education, } \\
\text { income }\end{array}$ & $\begin{array}{l}\text { PB: Poor general health } \\
\text { service utilisation, poor } \\
\text { access } \\
\text { SP: Most sought medical help } \\
\text { within } 2 \text { months }\end{array}$ & $\begin{array}{l}\text { PB: NR } \\
\text { SP: Longer time to SP } \\
\text { associated with lower income } \\
\text { for breast and colorectal } \\
\text { cancer }\left(x^{2}, p<0.05^{* *}\right) \text { and } \\
\text { lower education for all } \\
\text { tumour sites }\left(x^{2}, p<0.05^{* *}\right)\end{array}$ & Medium \\
\hline Schmid-Wendter (2002) [40] & $\begin{array}{l}\text { Retrospective } \\
\text { Quantitative }\end{array}$ & $\begin{array}{l}233 \text { men }(n=109) \\
\text { and women } \\
(n=109) . \text { Mean } \\
\text { age: } 54.5 \text { years }\end{array}$ & Germany & Melanoma & Education & $\begin{array}{l}\text { K: Previous knowledge of } \\
\text { melanoma, symptom } \\
\text { interpretation } \\
\text { B: Fear } \\
\text { PB: Lesion not visible, too } \\
\text { busy } \\
\text { SP: Sought medical help } \\
\text { within } 1 \text { month }(15.5 \%)\end{array}$ & $\begin{array}{l}\text { K: Higher education more } \\
\text { likely to have knowledge } \\
\text { about melanoma } \\
\left(x^{2}, p<0.001^{* *}\right) \\
\text { B: NR } \\
\text { PB: NR } \\
\text { SP: NR }\end{array}$ & Medium \\
\hline Siminoff et al. (2014) [35] & $\begin{array}{l}\text { Retrospective } \\
\text { Mixed methods }\end{array}$ & $\begin{array}{l}252 \text { men }(n=132) \\
\text { and women } \\
(n=120) \text {. Mean } \\
\text { age: } 58 \text { years } \\
\text { (range } 25 \text { to } 94 \\
\text { years) }\end{array}$ & US & Colorectal & $\begin{array}{l}\text { Education, } \\
\text { Employment, } \\
\text { Income }\end{array}$ & $\begin{array}{l}\text { K: Symptom interpretation } \\
(39.7 \% \text { did not think } \\
\text { symptom was serious) } \\
\text { PB: Financial barriers ( } 28.6 \%), \\
\text { fear of diagnostic tests } \\
(24.3 \%) \text {, embarrassment } \\
(11.9 \%) \\
\text { SP: Mean appraisal delay } \\
\text { (4.8 months) }\end{array}$ & $\begin{array}{l}\text { K: NR } \\
\text { PB: NR } \\
\text { SP: NS association between } \\
\text { time to SP and } \\
\text { socioeconomic group } \\
\text { (statistics NR) }\end{array}$ & Medium \\
\hline Simon et al. (2010) [49] & $\begin{array}{l}\text { Retrospective } \\
\text { Quantitative }\end{array}$ & $\begin{array}{l}236 \text { men }(n=968) \\
\text { and women } \\
(n=1240) .11 .4 \% \\
(n=236) \text { had } \\
\text { experienced a } \\
\text { symptom in the } \\
\text { past } 3 \text { months }\end{array}$ & UK & All & Occupation & $\begin{array}{l}\text { K: Recognition (better } \\
\text { knowledge if experienced a } \\
\text { symptom previously); } \\
\text { symptom interpretation } \\
\text { (worry symptom might be } \\
\text { cancer) } \\
\text { PB: Emotional and practical } \\
\text { barriers } \\
\text { SP: Symptom experience: } \\
11.4 \% \text { experienced symptom } \\
\text { in past } 3 \text { months ( } 75 \% \\
\text { consulted a GP about } \\
\text { symptom) }\end{array}$ & $\begin{array}{l}\text { K: NS association between } \\
\text { symptom interpretation and } \\
\text { socioeconomic group } \\
\text { (statistics NR) } \\
\text { PB: NR } \\
\text { SP: NS association between } \\
\text { SP and socioeconomic group } \\
\text { (statistics NR) }\end{array}$ & Good \\
\hline $\begin{array}{l}\text { Smith and Anderson } \\
\text { (1985) [51] }\end{array}$ & $\begin{array}{l}\text { Retrospective } \\
\text { Quantitative }\end{array}$ & $\begin{array}{l}82 \text { women. Age } \\
\text { range: } 20 \text { to } 54 \\
\text { years }\end{array}$ & US & Ovarian & $\begin{array}{l}\text { Income, } \\
\text { education, } \\
\text { occupation }\end{array}$ & $\begin{array}{l}\text { K: Symptom interpretation } \\
\text { ('cancer', } 10 \%) \\
\text { B: Fear } \\
\text { PB: Previous benign diagnosis } \\
\text { SP: Median time to symptom } \\
\text { presentation ( } 4 \text { weeks) }\end{array}$ & $\begin{array}{l}\text { K: NS association between } \\
\text { symptom interpretation and } \\
\text { socioeconomic group } \\
\text { (statistics NR) } \\
\text { B: NR }\end{array}$ & Medium \\
\hline
\end{tabular}

bowel/bladder habits $\left(x^{2}\right.$

$(1,1982)=56.87, p<0.001)$

cough $\left(x^{2}(1,1984)=48.32\right.$

$p<0.001)$, unexplained weight

loss $\left(x^{2}(1,1963)=77.73\right.$

$p<0.001)$ 
Table 1 Table of included studies (Continued)

\begin{tabular}{|c|c|c|c|c|c|c|c|c|}
\hline & & & & & & & $\begin{array}{l}\text { PB: NR } \\
\text { SP: NR }\end{array}$ & \\
\hline Temoshok et al. (1983) [75] & $\begin{array}{l}\text { Retrospective } \\
\text { Quantitative }\end{array}$ & $\begin{array}{l}106 \text { men and } \\
\text { women. Age } \\
\text { range: } 18 \text { to } \\
72 \text { years. }\end{array}$ & US & Melanoma & $\begin{array}{l}\text { Education, } \\
\text { occupation }\end{array}$ & $\begin{array}{l}\text { K: Previous knowledge of } \\
\text { melanoma } \\
\text { B: Melanoma not a serious } \\
\text { disease } \\
\text { PF: Lesion visible (face and } \\
\text { neck) } \\
\text { SP: Mean time to symptom } \\
\text { presentation (4 months) }\end{array}$ & $\begin{array}{l}\text { K: No association with } \\
\text { knowledge and occupation } \\
\text { (statistics NR) } \\
\text { B: NR } \\
\text { PF: NR } \\
\text { SP: No association with time } \\
\text { to SP and occupation } \\
\text { (statistics NR) }\end{array}$ & Poor \\
\hline Tod et al. (2008) [80] & $\begin{array}{l}\text { Retrospective } \\
\text { Qualitative }\end{array}$ & $\begin{array}{l}20 \text { men }(n=12) \\
\text { and women } \\
(n=8) .\end{array}$ & UK & Lung & Occupation & $\begin{array}{l}\text { K: Symptom interpretation } \\
\text { (poor, symptoms usually } \\
\text { interpreted as acute } \\
\text { conditions) } \\
\text { B: Fear, fatalism } \\
\text { PB: If previously given up } \\
\text { smoking (thought risk of lung } \\
\text { cancer was nil), worry about } \\
\text { the wasting doctors time, } \\
\text { previous bad experiences } \\
\text { with health system, blame, } \\
\text { stigma, stoicism, poor health } \\
\text { service utilisation } \\
\text { PF: Active encouragement } \\
\text { from family member } \\
\text { SP: Range in time to } \\
\text { symptom presentation } \\
\text { (0 to } 24 \text { months) }\end{array}$ & & Good \\
\hline Tomlinson et al. (2012) [60] & $\begin{array}{l}\text { Retrospective } \\
\text { Quantitative }\end{array}$ & $\begin{array}{l}87 \text { men }(n=56) \\
\text { and women } \\
(n=31) . \text { Mean } \\
\text { age: } 65 \text { years. }\end{array}$ & Canada & Colorectal & Education & $\begin{array}{l}\text { K: Symptom interpretation } \\
\text { PB: Self medication } \\
\text { SP: Waited over } 1 \text { month to } \\
\text { seek medical help ( } 51 \%)\end{array}$ & $\begin{array}{l}\text { K: NR } \\
\text { PB: NR } \\
\text { SP: NS association between } \\
\text { education and time to SP } \\
\left(x^{2}, p>0.05^{* *}\right)\end{array}$ & Medium \\
\hline Trivers et al. (2011) [52] & $\begin{array}{l}\text { Hypothetical } \\
\text { Quantitative }\end{array}$ & $\begin{array}{l}2991 \text { women. } \\
65 \% \text { were aged } \\
45 \text { years and over. }\end{array}$ & US & $\begin{array}{l}\text { Gynaeco- } \\
\text { logical }\end{array}$ & $\begin{array}{l}\text { Education, } \\
\text { Income }\end{array}$ & $\begin{array}{l}\text { B: Concern about developing } \\
\text { gynaecological cancer } \\
\text { PB: Being premenopausal } \\
\text { SP: } 50 \text { \% of women would } \\
\text { seek help for most symptoms }\end{array}$ & $\begin{array}{l}\text { B: NR } \\
\text { PB: NR } \\
\text { SP: NS association between } \\
\text { SP intentions and } \\
\text { socioeconomic group } \\
\text { (statistics NR) }\end{array}$ & Medium \\
\hline Van Osch et al. (2007) [48] & $\begin{array}{l}\text { Hypothetical } \\
\text { Quantitative }\end{array}$ & $\begin{array}{l}459 \text { men }(49 \%) \\
\text { and women } \\
\text { (51\%) over the } \\
\text { age of } 55 . \text { Mean } \\
\text { age: } 68.6 \text { years. }\end{array}$ & Netherlands & All & Education & $\begin{array}{l}\text { K: Recognition (low to } \\
\text { moderate, mean: 6.2) } \\
\text { B: Benefits of early detection } \\
\text { SP: Fair. Inconsistent for } \\
\text { urgent symptoms, good for } \\
\text { prolonged symptoms }\end{array}$ & $\begin{array}{l}\text { K: NR } \\
\text { B: NR } \\
\text { SP: Lower education } \\
\text { associated with shorter time } \\
\text { to SP }(F(2,436)=6.084 \text {, } \\
p<0.01)\end{array}$ & Good \\
\hline
\end{tabular}


Table 1 Table of included studies (Continued)

\begin{tabular}{|c|c|c|c|c|c|c|c|c|}
\hline Waller et al. (2009) [53] & $\begin{array}{l}\text { Hypothetical } \\
\text { Quantitative }\end{array}$ & $\begin{array}{l}1500 \text { men and } \\
\text { women from } \\
\text { various ethnic } \\
\text { minority groups. }\end{array}$ & England & All & Occupation & $\begin{array}{l}\text { K: Recall (poor, mean: 1.2) } \\
\text { and recognition (poor, } \\
\text { mean: 4.7) } \\
\text { PB: Worry what doctor might } \\
\text { find (most endorsed) } \\
\text { SP: African and Caribbean } \\
\text { groups anticipated fastest } \\
\text { time to symptom } \\
\text { presentation }\end{array}$ & $\begin{array}{l}\text { K: Higher socioeconomic } \\
\text { group associated with higher } \\
\text { recall }(F(1,1487)=6.12, p< \\
0.01) \text { and higher recognition } \\
(F(1,1487)=5.45, p<0.05) \\
\text { PB: NR } \\
\text { SP: NR }\end{array}$ & Good \\
\hline Walter et al. (2014) [41] & $\begin{array}{l}\text { Retrospective } \\
\text { Qualitative }\end{array}$ & $\begin{array}{l}63 \text { men }(n=31) \\
\text { and women } \\
(n=32) . \text { Age } \\
\text { range: } 29-93 \text { years. }\end{array}$ & UK & Melanoma & Education & $\begin{array}{l}\text { K: Symptom attributions } \\
\text { (initially attributed to benign } \\
\text { skin conditions or normal } \\
\text { life changes) } \\
\text { PB: Worry about wasting the } \\
\text { doctors time, service barriers, } \\
\text { competing life priorities, } \\
\text { reassurance following } \\
\text { symptom disclosure } \\
\text { PF: Family history of } \\
\text { melanoma, perceptions of } \\
\text { high risk, symptom } \\
\text { disclosure, symptom noticed } \\
\text { by another person } \\
\text { SP: Range 1-303 weeks }\end{array}$ & & Good \\
\hline Whitaker et al. (2014) [55] & $\begin{array}{l}\text { Retrospective } \\
\text { Quantitative }\end{array}$ & $\begin{array}{l}1724 \text { men } \\
(n=789) \text { and } \\
\text { women }(n=921) \\
\text { over the age of } \\
\text { 50. Mean age: } \\
64.4 \text { years. }\end{array}$ & England & All & $\begin{array}{l}\text { Postcode, } \\
\text { education, } \\
\text { employment }\end{array}$ & $\begin{array}{l}\text { K: Symptom interpretations } \\
\text { ( } 2 \% \text { thought symptom was } \\
\text { cancer, highest interpretation } \\
\text { for 'unexplained lump'), } \\
\text { perceived seriousness of } \\
\text { symptoms } \\
\text { SP: Symptom experience } \\
\text { ( } 53 \% \text { experienced at least } 1 \\
\text { symptom in past } 3 \text { months). } \\
59 \% \text { contacted GP about } \\
\text { symptom }\end{array}$ & 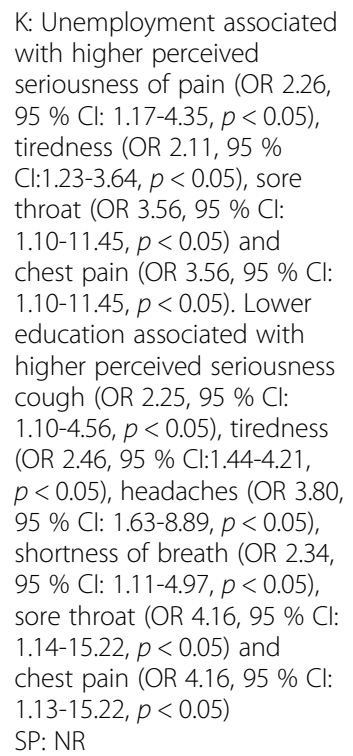 & Good \\
\hline
\end{tabular}

eassurance following

symptom disclosure

disclosure, symptom noticed

SP. Range 1-303 weeks

$5 \%$ Cl: 1.17-4.35, $p<0.05)$

tiredness (OR 2.11, $95 \%$

throat (OR 3.56, $95 \%$ Cl:

$p<0.05)$, tiredness

13-15.22, $p<0.05)$ 
Table 1 Table of included studies (Continued)

Whitaker et al. (2015) [81] Retrospective

Whitaker et al. (2015) [81]

$\begin{array}{ll}\text { Retrospective } & 48 \text { men }(n=23) \\ \text { Qualitative } & \text { and women } \\ & (n=25) \text { over the } \\ & \text { age of 50. Mean } \\ & \text { age: } 64.4 \text { years. }\end{array}$

England

All

Education,
employment

$\mathrm{K}$ : Symptom interpretations

symptom

associated with cancer)

PB: Stoicism, fear of

diagnostic tests, worry about

wasting doctors time, service

barriers, negative attitudes

towards HCPs, medical

mistrust

PF: Development of new

symptoms, persistence of

symptoms, symptonce of

symptoms, symptom

disclosure, fea

$33.3 \%$ contacted GP with

'persistent cough', $100 \%$

contacted GP with

'unexplained bleeding'

$K=$ cancer symptom knowledge; $B=$ beliefs about cancer; $P B=$ perceived barriers to symptom presentation; $P F=$ perceived facilitators to symptom presentation; $S P=$ time to symptom presentation; $N R=$ not reported; $N S=$ not significant; ${ }^{*}$-value not reported; **other statistics not reported 
Studies which measured actual time to symptom presentation reported the longest delays in symptom presentation among individuals with lower educational attainment [33, 34, 42, 54, 58-61], lower annual income $[61,62]$, lower occupation and employment $[43,61,63]$ and those from deprived areas [64]. This effect was also observed in studies of actual symptom presentation where multiple socioeconomic indices were reported $[34,42,44,61,65,66]$. Twenty-two studies found no group differences for socioeconomic group indicators and time to symptom presentation $[30,33,35-37,45$, $46,49,50,52,60,67-77]$.

\section{Knowledge}

Knowledge of symptoms based on recall methods was generally lower than in studies that used recognition methods. Lump symptoms were the most recalled and well-recognised potential cancer symptom [19, 32, 48, $50,53,56,64,78]$. This was supported by retrospective studies where patients presenting with a lump were most likely to have attributed their lump symptom to cancer [39, 43, 45, 74, 79]. Knowledge was generally poor for non-specific symptoms of cancer. Symptoms such as fatigue or unexplained weight loss were poorly recalled or recognised as potential symptoms of cancer [28, 29, 31, 53, 78]. Poorer cancer symptom knowledge was associated with lower socioeconomic group when measured by educational attainment $[28,32,40,50,54,56]$, occupation [53] and multiple indicators [19, 28, 66]. These findings were consistent across site-specific and non site-specific studies, suggesting poor general cancer symptom knowledge in lower socioeconomic groups regardless of cancer type.

In retrospective studies, patients experiencing nonspecific symptoms recalled attributing them to other benign causes or life stresses $[35,51,55,65,69,76$, $80,81]$ or not recognising the seriousness of their symptoms $[9,33,35,37,40,42,43,45,47,51,54$, $55,57,60,65,68,76,77,81,82]$ resulting in patients delaying symptom presentation $[35,39,51,76]$ or later stage at diagnosis [69].

\section{Beliefs about cancer}

In most studies, beliefs were formed from participants' past experiences of cancer, usually witnessing friends or family with the disease [36, 43, 47, 59, 78, 79]. Positive beliefs were identified in nine studies [30, 36, 43, 48, 54, $58,78,79]$ and tended to focus on the effectiveness of modern cancer treatments, where participants expressed trust in doctors and the medical system and endorsed the benefits of early diagnosis $[30,58,59,78]$ or acknowledged that cancer can be cured [78]. Such beliefs tended to encourage timely symptom presentation to a primary care physician $[30,58,59,78,79]$. One study found that those with lower educational attainment were less likely to endorse positive beliefs about the benefits of early detection [54].

Negative beliefs tended to manifest in fear or fatalism regarding cancer. Fear was frequently reported across all studies examining beliefs. This included fear of diagnosis $[34,39,58,63,74,80,81,83]$, fear of treatment [30, 43, $57-59,68,78,83]$ and fear of dying [59, 78, 83]. Fatalistic beliefs were a common theme throughout studies, but were expressed only by a minority of participants per study [34, 36, 42, 56, 59, 61, 78, 79, 84]. Fearful and fatalistic beliefs about cancer were more likely to be expressed by individuals from lower socioeconomic groups based on educational attainment $[36,50]$ or multiple indices [42, 71].

When considering time to symptom presentation, fearful beliefs about cancer appeared to operate at the two extremes of immediate or prolonged symptom presentation. For participants whose fearful beliefs encouraged immediate (actual or hypothetical) presentation to doctors $[43,45,58,59,61,74,78,79,84]$, a visit to doctors was used to alleviate anxiety associated with the symptom [43, 47, 58, 59, 61, 77, 78]. This was usually coupled with the participant expressing trust in the medical profession and positive beliefs surrounding early diagnosis [43, 59].

For individuals whose fearful beliefs led to prolonged delays (sometimes years) [30, 34, 38, 39, 43, 47, 51, 61, $68,74,78,79]$, denial of or ignoring symptoms initially alleviated anxiety associated with the symptom [38, 39, 47, 59, 68, 72, 76, 78, 79]. Such beliefs were usually combined with fatalistic beliefs such as 'cancer cannot be cured' $[59,61,79]$, and were associated with the longest times to symptom presentation or were expressed by those with advanced stage disease [36, 56, 59, 84]. This is likely to reflect a lack of perceived benefit in presenting to doctors due to the belief that 'nothing can be done' $[59,78]$.

\section{Barriers to symptom presentation}

Some participants reported service barriers relating to concerns about wasting doctors' time [19, 29, 31, 34, 41, $43,55,80,81]$, lack of continuity with primary care doctor $[42,81]$ or difficulties with accessing and making an appointment [29, 32, 34, 38, 53, 55, 56, 65, 78, 81]. For others, practical barriers such as being 'too busy to make an appointment' were reported and these delayed symptom presentation $[31,39,40,43,49,74,77,78]$. Low general health service utilisation for acute or long term conditions lengthened time to cancer symptom presentation $[9,34,42,43,57,58,61,66,68,73,77,78$, 80, 84]. Emotional barriers included embarrassment or fear associated with undergoing intimate diagnostic tests $[19,29,31,34,35,49,57,78,81]$. 
Practical barriers such as 'being too busy' were more frequently reported in high socioeconomic groups [19]. In countries where patients pay for their healthcare, those with lower annual income were more likely to report the cost of a consultation as a barrier to symptomatic presentation $[38,63]$.

\section{Facilitators to symptom presentation}

The most common facilitator of symptom presentation was disclosure of symptoms to a family member or friend $[34,39,41,43,45,47,55,61,65,70,73,76-79$, $81,84,85]$. In some cases, this reduced time to symptom presentation by half [36] or by six times [45]. The appearance of a new symptom $[43,69,76,83]$ or persistence of the current symptom $[45,69,76,81,84]$ facilitated decisions to seek medical help. One study found that individuals from a lower socioeconomic group who disclosed their symptom to a family member or friend took longer to seek medical help compared to those from a higher socioeconomic group [65]. In five studies, participants waited until they developed another health complaint or tagged their cancer symptom on to the end of a consultation which provided an opportunity to disclose the cancer symptom during the consultation $[42,45,68,81,82]$.

\section{Discussion}

This review is the first to systematically explore how knowledge, beliefs and barriers/facilitators to symptom presentation affect actual or anticipated cancer symptom presentation in relation to socioeconomic group, across all tumour sites. Poor knowledge of non-specific cancer symptoms such as fatigue and weight loss prolonged presentation due to misattribution of symptoms in lower socioeconomic groups. In contrast, lump and bleeding symptoms were most frequently recalled, recognised and prompted the fastest symptom presentation. A knowledge gradient was observed, where poorer cancer symptom knowledge was associated with lower socioeconomic group based on multiple indices. There was some evidence to suggest that those from a lower socioeconomic group were more likely to hold fearful and fatalistic beliefs about cancer and less likely to endorse positive beliefs about the benefits of early diagnosis. Such combinations of fearful and fatalistic beliefs were associated with prolonged symptom presentation. In addition, emotional barriers to symptom presentation such as worry what the doctor might find were more likely to be endorsed in lower socioeconomic groups. Such poor knowledge and prevalent beliefs might account for the long actual delays and later stage cancers diagnosed in lower socioeconomic groups. Disclosure of a symptom to a family member or friend was a key facilitator in the decision to seek medical help, although there was some evidence to suggest that symptom disclosure acted as a barrier in lower socioeconomic groups.

Most included studies were of medium quality. In many studies, socioeconomic group was measured but not reported for all outcome variables. Most studies only reported socioeconomic group differences for symptom presentation. Twenty-three studies reported socioeconomic group differences for the other outcome measures: knowledge, beliefs and barriers/facilitators to symptom presentation. A further eight studies could have met the inclusion criteria, but were excluded due to non-reporting of any outcomes associated with socioeconomic group [14, 86-92]. Methodological limitations included a long duration between cancer diagnosis and participation in retrospective studies, and samples biased towards higher socioeconomic groups. In some studies, socioeconomic variation was insufficient to perform statistical analysis on all outcomes.

There are methodological limitations associated with retrospective (actual symptom presentation) and hypothetical (anticipated symptom presentation) designs. Whilst retrospective studies are affected by recall bias, hypothetical studies rely on intentions which may not translate into actual presentation behaviour [93]. This was observed in the variation between actual and hypothetical time to symptom presentation, where participants anticipated prompt symptom presentation but in reality reported longer delays. Study designs exploring actual symptom presentation behaviour in a population sample are likely to reduce some of the limitations associated with retrospective and hypothetical symptom presentation study designs. In such study designs, participants disclose actual symptoms experienced in the past three months, usually prompted by a list (without any mention of cancer), and reasons for not consulting a doctor are explored $[49,54,55,81]$.

The limitations of this review include problems relating to retrieval of studies and analysis of the evidence. Due to poor indexing of studies in this topic area under the $\mathrm{MeSH}$ indexing in this topic area, a high proportion of studies $(n=11)$ was found through hand-searching. Additionally, meta-analysis was precluded by the wide range of qualitative and quantitative data collection methods of included studies. Finally, other factors such as age, gender and ethnicity can affect symptom presentation [6, 18]; however, interactions between these variables and socioeconomic group were not addressed in the current review.

The findings of the current review confirm that failure to appreciate the seriousness of symptoms $[6,16]$ and non-disclosure of symptoms $[6,15]$ lengthened time to symptom presentation. Our findings accord with previous studies in which negative beliefs [20], longer time to 
actual symptom presentation [6] and low suspicion for cancer symptoms [94] were associated with low socioeconomic group [6]. The current findings support Mitchell et al.'s (2008) [16] review of colorectal cancer patients, in which fear of cancer either lengthened or shortened time to symptom presentation. Such findings might be explained by Type I and Type II information processing systems. Type I processing is a fast and automatic system, which represents an individual's 'gut reaction' to an event, whereas Type II is a slower, more thoughtful and deliberative system [95]. Whilst most people initially experience fear in reaction to a worrying symptom (Type I processing), cognitions during Type II processing may influence the decision to seek medical help since these are slower and may help someone to rationalise the situation [96]. If an individual has had time to consider the benefits of seeking medical help, and based upon their previous beliefs about early diagnosis, such beliefs may override the Type 1 fear response. We found evidence to suggest a higher prevalence of fearful and fatalistic beliefs in lower socioeconomic groups and some evidence for fewer positive beliefs surrounding the benefits of early diagnosis in lower socioeconomic groups. This suggests that Type I beliefs may not be overridden by Type 2 responses relating to the benefits of early diagnosis due to lower knowledge or higher emotive responses. As a consequence this may delay symptom presentation. Findings relating to symptom disclosure suggest that people use the 'lay system' of healthcare (consulting family and friends) before making the decision to access formal healthcare [13, 97, 98]. However, among individuals from low socioeconomic groups, disclosing symptoms to someone with equally poor knowledge and Type I negative automatic beliefs about cancer may encourage false reassurance in the benign nature of symptoms and consequently no urgency to seek medical help.

Cancer awareness interventions should be carefully developed to target those who are most likely to present with advanced stage disease: lower socioeconomic groups with low symptom knowledge and fearful and fatalistic beliefs about cancer. Such an intervention should utilise an individual's social networks to facilitate distribution of information [97], highlighting the significance of non-lump symptoms as potentially indicative of cancer, along with advice on an appropriate time in which an individual should seek medical help and how to access such help [99]. This should be coupled with information outlining the benefits of early diagnosis and improved effectiveness of modern treatments for cancer, countering negative beliefs surrounding cancer. Future research should evaluate the effectiveness of such interventions in lower socioeconomic groups.

\section{Conclusion}

Knowledge of potential cancer symptoms, beliefs about cancer and barriers to symptom presentation work in combination to influence symptom presentation: knowledge is necessary for accurate symptom appraisal, but beliefs about cancer and barriers to symptom presentation influence the decision to seek medical help or not. This is especially important in the context of socioeconomic deprivation, where lower knowledge, higher negative beliefs about cancer and perceived barriers may lead to avoidable delays, later stage of diagnosis and ultimately poorer survival outcomes. Targeted interventions should not only educate people about symptoms for cancer, but also work to break down unhelpful myths surrounding cancer survival and treatment options. They should address the barriers that people in lower socio-economic groups experience, and use social networks to raise awareness and support early symptom presentation.

\section{Additional file}

Additional file 1: Appendix 1. Search terms. (DOCX $13 \mathrm{~kb}$ )

\section{Abbreviations}

NAEDI: National awareness and early diagnosis initiative; SPIDER: Sample phenomenon of interest, design, evaluation, research type; CASP: Critical appraisal skills programme; OECD: Organisation for economic co-operation and development.

\section{Competing interests}

The authors declare that they have no competing interests.

\section{Authors' contributions}

GMM, KEB, AGE and FW were responsible for the concept and design and conduct of the study. AGE gave additional advice on methodology. GMM was responsible for collection of data and manuscript preparation. RR was responsible for double checking at all stages of the search. KEB, AGE and FW extensively reviewed and edited the manuscript drafts. All authors were involved in interpretation of results and approved the final version of the manuscript.

\section{Acknowledgements}

The authors would like to thank Dr Stephanie Smits for her contribution to interpretation of data from quantitative studies. This study was funded by Tenovus Cancer Care.

Received: 20 January 2015 Accepted: 3 December 2015

Published online: 23 December 2015

\section{References}

1. Ellis L, Coleman M, Rachet B. How many deaths would be avoidable if socioeconomic inequalities in cancer survival in England were eliminated? A national population-based study, 1996-2006. Eur J Cancer. 2012;48(2):270-8.

2. McPhail S, Johnson S, Greenberg D, Peake M, Rous B. Stage at diagnosis and early mortality from cancer in England. Br J Cancer. 2015;112:S108-15.

3. Rachet B, Ellis L, Maringe C, Chu T, Nur U, Quaresma M, et al. Socioeconomic inequalities in cancer survival in England after the NHS cancer plan. Br J Cancer. 2010;103(4):446-53.

4. Woods LM, Rachet B, Coleman MP. Orogins of socio-economic inequalities in cancer survival: a review. Ann Oncol. 2006;17(1):5-19. 
5. Rutherford M, Ironmonger L, Ormiston-Smith N, Abel G, Greenberg D, Lyratzopoulos $\mathrm{G}$, et al. Estimating the potential survival gains by eliminating socioeconomic and sex inequalities in stage at diagnosis of melanoma. $\mathrm{Br} J$ Cancer. 2015;112:S116-23.

6. Macleod U, Mitchell E, Burgess C, Macdonald S, Ramirez A. Risk factors for delayed presentation and referral of symptomatic cancer: evidence for common cancers. British J Cancer. 2009;101:S92-S101.

7. Lyratzopoulos G, Abel GA, Brown CH, Rous BA, Vernon SA, Roland M, et al. Socio-demographic inequalities in stage of cancer diagnosis: evidence from patients with female breast, lung, colon, rectal, prostate, renal, bladder, melanoma, ovarian and endometrial cancer. Ann Oncol. 2013;24(3):843-50.

8. Allgar V, Neal R. Delays in the diagnosis of six cancers: analysis of data from the National Survey of NHS Patients: Cancer. Br J Cancer. 2005;92(11):1959-70.

9. Ristvedt S, Trinkaus K. Psychological factors related to delay in consultation for cancer symptoms. Psycho-Oncol. 2005;14(5):339-50.

10. Lyratzopoulos G, Saunders C, Abel G, McPhail S, Neal R, Wardle J, et al. The relative length of the patient and the primary care Interval in patients with 28 common and rarer cancers. Br J Cancer. 2015;112:S35-40.

11. Walter F, Webster A, Scott S, Emery J. The Andersen Model of Total Patient Delay: a systematic review of its application in cancer diagnosis. J Health Serv Res Policy. 2012;17(2):110-8.

12. Whitaker $\mathrm{K}, \mathrm{Scott} \mathrm{S}$, Wardle J. Applying symptom appraisal models to understand sociodemographic differences in responses to possible cancer symptoms: a research agenda. Br J Cancer. 2015;112:S27-34.

13. Low E, Whitaker K, Simon A, Sekhon M, Waller J. Women's interpretation of and responses to potential gynaecological cancer symptoms: a qualitative interview study. BMJ Open. 2015;5(7), e008082.

14. Sheikh I, Ogden J. The role of knowledge and beliefs in help seeking behaviour for cancer: a quantitative and qualitative approach. Patient Educ Couns. 1998;35(1):35-42.

15. Bish A, Ramirez A, Burgess C, Hunter M. Understanding why women delay in seeking help for breast cancer symptoms. J Psych Res. 2005;58(4):321-6.

16. Mitchell E, Macdonald S, Campbell N, Weller D, Macleod U. Influences on pre-hospital delay in the diagnosis of colorectal cancer: a systematic review. Br J Cancer. 2008;98(1):60-70.

17. Smith L, Pope C, Botha J. Patients' help-seeking experiences and delay in cancer presentation: a qualitative synthesis. Lancet. 2005;366(9488):825-31.

18. Hiom S. Diagnosing cancer earlier: reviewing the evidence for improving cancer survival. Br J Cancer. 2015;112:S1-5.

19. Robb K, Stubbings S, Ramirez A, Macleod U, Austoker J, Waller J, et al. Public awareness of cancer in Britain: a population-based survey of adults. Br J Cancer. 2009;101:S18-23.

20. Quaife S, Winstanley K, Robb K, Simon A, Ramirez A, Forbes L, et al. Socioeconomic inequalities in attitudes towards cancer: an international cancer benchmarking partnership study. Eur J Cancer Prev. 2015;24(3):253-60

21. Ramirez A, Westcombe A, Burgess C, Sutton S, Littlejohns P, Richards M. Factors predicting delayed presentation of symptomatic breast cancer: a systematic review. Lancet. 1999;353(9159):1127-31.

22. Moher D, Liberati A, Tetzlaff J, Altman D, The PRISMA. Group. Preferred Reporting Items for Systematic Reviews and Meta-Analysis: The PRISMA Statement. Plos Med. 2009:6(7), e1000097.

23. McCutchan G, Wood F, Edwards A, Richards, R, Brain K A. Systematic review of cancer awareness, beliefs about cancer and symptomatic presentation in the context of social deprivation. 2014. CRD42014013220 Available from: http://www.crd.york.ac.uk/PROSPERO/display_record. asp?ID=CRD42014013220

24. Cooke A, Smith D, Booth A. Beyond PICO: The SPIDER Tool for Qualitative Evidence Synthesis. Qual Health Res. 2012;22(10):1435-43.

25. OECD. http://www.oecd.org/about/membersandpartners/list-oecd-membercountries.htm (2014). Accessed: 6th January 2014.

26. Popay J, Roberts H, Sowden A, Petticrew M, Arai L, Rodgers M, et al. Guidance on the conduct of narrative synthesis in systematic reviews: A product from the ESRC Methods Programme. Lancaster: Lancaster University; 2006.

27. CASP. http://www.casp-uk.net/\#!casp-tools-checklists/c18f8 (2014). Accessed 13th May 2014.

28. Brain KE, Smits S, Simon AE, Forbes $L$, Roberts C, Robbe IJ, et al. Ovarian cancer symptom awareness and anticipated delayed presentation in a population sample. BMC Cancer. 2014;14:171-81. do:10.1186/1471-2407-14-17.
29. Forbes L, Atkins L, Thurnham A, Layburn J, Haste F, Ramirez A. Breast cancer awareness and barriers to symptomatic presentation among women from different ethnic groups in East London. Br J Cancer. 2011;105(10):1474-9.

30. Hunter MS, Grunfield EA, Ramirez AJ. Help-seeking intentions for breastcancer symptoms: a comparison of the self-regulation model and the theory of planned behaviour. Br J Health Psychology. 2003;8(3):319-33.

31. Low E, Waller J, Menon U, Jones A, Reid F, Simon A. Ovarian cancer symptom awareness and anticipated time to help-seeking for symptoms among UK women. J Fam Plan R Health. 2013;39(3):163-71.

32. Quaife S, Forbes L, Ramirez A, Brain K, Donnelly C, Simon A, et al. Recognition of cancer warning signs and anticipated delay in help-seeking in a population sample of adults in the UK. Br J Cancer. 2014;110(1):12-8.

33. Ristvedt SL, Pruitt SL, Trinkhaus KM. Appraisal of emerging symptoms of colorectal cancer: associations with dispositional, demographic, and tumour characteristics. J Behav Med. 2014;37(4):698-708. doi:10.1007/s10865-013-9519-4.

34. Kakagia D, Trypsiannis G, Karanikas M, Mitrakas A, Lyratzopoulos N, Polychronidis A. Patient-Related Delay in Presentation for Cutaneous Squamous Cell Carcinoma. A Cross-Sectional Clinical Study. Onkologie. 2013;36(12):738-44.

35. Siminoff $L$, Thomson M, Dumenci L. Factors associated with delayed patient appraisal of colorectal cancer symptoms. Psycho-Oncol. 2014;23(9):981-8.

36. Chonjnacka-Szwalowska G, Koscielak R, Karasiewicz K, Majkowicz M, Kozaka J. Delays in seeking cancer diagnosis in relation to beliefs about the curability of cancer in patients with different disease locations. Psychol Health. 2013;28(2):154-70.

37. Oliveria SA, Christos PJ, Halpern AC, Fine JC, Barnhill RL, Berwick M. Patient knowledge, awareness, and delay in seeking medical attention for malignant melanoma. J Clin Epidemiol. 1999;52(11):1111-6.

38. Freidman LC, Kalidas M, Elledge R, Dulay MF, Romero C, Chang J, et al. Medical and psychological predictors of delay in seeking medical consultation for breast symptoms in women in a public sector setting. J Behav Med. 2006;29(4):327-34.

39. Gould J, Fitzgerald B, Fergus K, Clemons M, Baig F. Why women delay seeking assistance for locally advanced breast cancer. Can Oncol Nurs J. 2010;20(1):23-9.

40. Schmid-Wendtner M, Baumert J, Stange J, Volkenandt M. Delay in the diagnosis of cutaneous melanoma: an analysis of 233 patients. Melanoma Res. 2002;12(4):389-94.

41. Walter F, Birt L, Cavers D, Scott S, Emery J, Burrows N, Cavanagh G, MacKie R, Weller D, Campbell C. 'This isn't what mine looked like': a qualitative study of symptom appraisal and help seeking in people recently diagnosed with melanoma. BMJ Open. 2014;4(7). doi:10.1136/bmjopen-2014-005566

42. Coates R, Bransfield D, Wesley M, Hankey B, Eley J, Greenberg R, et al. Differences between black and white women with breast cancer in time from symptom recognition to medical consulation. J Nat Cancer Inst. 1992;84(12):938-50.

43. Burgess $C$, Hunter M, Ramirez A. A qualitative study of delay among women reporting symptoms of breast cancer. Br J Gen Pract. 2001;51(473):967-71.

44. Caplan L. Patient delay in seeking help for potential breast cancer. Public Health Rev. 1995;23(3):263-74.

45. Burgess C, Ramirez A, Richards M, Love S. Who and what influences delayed presentation in breast cancer? Br J Cancer. 1998;77(8):1343-8.

46. Meechan G, Collins J, Petrie KJ. The relationship of symptoms and psychological factors to delay in seeking medical care for breast symptoms. Prev Med. 2003;36(3):374-8.

47. O'Mahony M, Hegarty J. Factors influencing women in seeking help from a health care professional on self discovery of a breast symptom, in an Irish context. J Clin Nurs. 2009:18(14):2020-9.

48. van Osch L, Lechner L, Reubsaet A, de Nooijer J, de Vries H. Passive cancer detection and medical help seeking for cancer symptoms: (in)adequate behavior and psychosocial determinants. Eur J Cancer Prev. 2007;16(3):266-74.

49. Simon A, Waller J, Robb K, Wardle J. Patient Delay in Presentation of Possible Cancer Symptoms: The Contribution of Knowledge and Attitudes in a Population Sample from the United Kingdom. Cancer Epidemiol Biomarkers Prev. 2010;19(9):2272-7.

50. McCaffery K, Wardle J, Waller J. Knowledge, attitudes, and behavioral intentions in relation to the early detection of colorectal cancer in the United Kingdom. Prev Med. 2003;36(5):525-35.

51. Smith EM, Anderson B. The effects of symptoms and delay in seeking diagnosis on stage of disease at diagnosis on stage of disease at diagnosis among women with cancers of the ovary. Cancer. 1985;56(11):2727-32. 
52. Trivers KF, Rodriguez JL, Hawkins NA, Cooper CP, Polonec L, Gelb CA. Intention to seek care for symptoms associated with gynaecologic cancers, HealthStyles survey, 2008. Prev Chronic Dis. 2011;8(6):1-9.

53. Waller J, Robb K, Stubbings S, Ramirez A, Macleod U, Austoker J, et al. Awareness of cancer symptoms and anticipated help seeking among ethnic minority groups in England. Br J Cancer. 2009;101:S24-30.

54. Cockburn J, Paul C, Tzelepis F, McElduff P, Byles J. Delay in seeking advice for symptoms that potentially indicate bowel cancer. Am J Health Behav. 2003;27(4):401-7

55. Whitaker K, Scott S, Winstanley K, Macleod U, Wardle J. Attributions of Cancer 'Alarm' Symptoms in a Community Sample. Plos One. 2014;9(12):e114028.

56. Facione N, Miaskowski C, Dodd M, Paul S. The self-reported likelihood of patient delay in breast cancer: New thoughts for early detection. Prev Med. 2002;34(4):397-407.

57. Fitzpatrick P, Corcoran N, Fitzpatrick J. Prostate cancer: how aware is the public? Br J Urology. 1998:82(1):43-8.

58. Cameron A, Hinton J. Delay in seeking treatment for mammary tumours. Cancer. 1968;21(6):1121-6.

59. Facione NC, Facione PA. The cognitive structuring of patient delay in breast cancer. Soc Sci Med. 2006;63(12):3137-49.

60. Tomlinson C, Wong C, Au HJ, Schiller D. Factors associated with delays to medical assessment and diagnosis for patients with colorectal cancer. Can Fam Physician. 2012;58:495-501.

61. Goldsen RK, Gerhardt PR, Handy VH. Some factors related to patient delay in seeking diagnosis for cancer symptoms. Cancer. 1957;10(1):1-7.

62. Samet JM, Hunt W, Lerchen ML, Goodwin JS. Delay in seeking care for cancer symptoms: a population-based study of elderly New Mexicans. J Natl Cancer Inst. 1988;80(6):432-8.

63. Lam WW, Fielding R, Chan R, Or A. Factors influencing delayed presentation with symptomatic breast cancer in Hong Kong Chinese women. Hong Kong Med J. 2009;15(3):4-7.

64. Forbes $L J$, Warburton F, Richards MA, Ramirez AJ. Risk factors for delay in symptomatic presentation: a survey of cancer patients. Br J Cancer. 2014;111(3):581-8. doi:10.1038/bjc.2014.304

65. Li WW, Lam WW, Wong JH, Chui A, Chan M, Or A, et al. Waiting to see the doctor: understanding appraisal and utilization components of consultation delay for new breast symptoms in Chinese women. Pyshcooncology. 2012;21(12):1316-23. doi:10.1002/pon.2038.

66. Rauscher GH, Ferrans CE, Kaiser K, Campbell RT, Calhoun EE, Warnecke RB. Misconceptions about breast lumps and delayed medical presentation in urban breast cancer patients. Cancer Epidemiol Biomarkers Prev. 2010;19(3):640-7. doi:10.1158/1055-9965.EPI-09-0997.

67. Burgess CC, Ramirez AJ, Smith P, Richards MA. Do adverse life events and mood disorders influence delayed presentation of breast cancer? J Psychosom Res. 2000:48(2):171-5.

68. Greer S. Psychological aspects: delay in treatment of breast cancer. Proc R Soc Med. 1974;67(6):470-3.

69. Carter-Harris L, Hermann CP, Draucker CB. Pathways to lung cancer diagnosis. J Am Assoc Nurse Pract. 2015. doi:10.1002/2327-6924.12242.

70. Esteva M, Leiva A, Ramos M, Pita-Fernandez S, Gonzalez-Lujan L, Casamitjana $\mathrm{M}$, et al. Factors related with symptom duration until diagnosis and treatment of symptomatic colorectal cancer. BMC Cancer. 2013;13(87). doi:10.1186/1471-2407-13-87.

71. Loehrer PJ, Greger HA, Weinberger M, Musick B, Miller M, Nichols C, et al Knowledge and beliefs about cancer in a socioeconomically disadvantaged population. Cancer. 1991;68(7):1665-7.

72. Magarey CJ, Todd PB, Blizard PJ. Psycho-social factors influencing delay and breast self-examination in women with symptoms of breast cancer. Soc Sci Med. 1977;11(4):229-32.

73. Rozniatowski O, Reich M, Mallet Y, Penel N, Fournier C, Lefebvre JL. Psychosocial factors involved in delayed consultations by patients with head and neck cancer. Head Neck. 2005;27(4):274-80. doi:10.1002/hed.20157.

74. Mor V, Masterson-Allen S, Goldberg R, Guadagnoli E, Wool MS. Pre-diagnostic symptom recognition and help seeking among cancer patients. J Community Health. 1990;15(4):253-66.

75. Temoshok L, DiClemente RJ, Sweet DM, Blois MS, Sagebiel RW. Factors related to patient delay in seeking medical attention for cutaneous malignant melanoma. Cancer. 1983;54(12):3048-53.

76. Brouha XD, Tromp DM, Hordijk G, Winnubst JA, de Leeuw R. Oral and pharyngeal cancer: analysis of patient delay at different tumour stages. Head Neck. 2005;27(11):939-45.
77. Richard MA, Grob JJ, Avril MF, Delaunay M, Gouvernet J, Wolkenstein P, et al. Delays in diagnosis and melanoma prognosis (I): the role of patients. Int J Cancer. 2000;89(3):271-9.

78. Marlow LA, McGregor LM, Nazaroo JY, Wardle J. Facilitators and barriers to help-seeking for breast and cervical cancer symptoms: a qualitative study with an ethnically diverse sample in London. Psychooncology. 2014;23(7):749-57. doi:10.1002/pon.3464.

79. O'Mahony M, Hegarty J, McCarthy G. Women's help seeking behaviour for self-discovered breast cancer symptoms. Eur J Oncol Nurs. 2011;15(5):410-8. doi:10.1016/j.ejon.2010.10.011.

80. Tod AM, Craven J, Allmark P. Diagnostic delay in lung cancer: a qualitative study. J Adv Nurs. 2008;61(3):336-43. doi:10.1111/j.1365-2648.2007.04542.x.

81. Whitaker KL, Macleod U, Winstanley K, Scott SE, Wardle J. Help seeking for cancer 'alarm' symptoms: a qualitative interview study of primary care patients in the UK. Br J Gen Pract. 2015;65(631):e96-e105.

82. Grant E, Silver K, Bauld R, Day R, Warnakulasuria S. The experiences of young oral cancer patients in Scotland: symptom recognition and delays in seeking professional help. Br Dent J. 2010;208(10):465-71. doi:10.1038/sj.bdj.2010.450

83. Facione NC, Dodd MJ. Women's narratives of help seeking for breast cancer Cancer Pract. 1995:3(4):219-25.

84. Facione NC, Dodd MJ, Holzemer WM, Meleis Al. Help-seeking for selfdiscovered breast symptoms. Implications for early detection. Cancer Pract. 1997;5(4):220-7.

85. Pedersen AF, Olesen F, Hansen R, Hansen RP, Zachariae R, Vedsted P. Social support, gender and patient delay. Br J Cancer. 2011;104(8):1249-55.

86. Brandner S, Muller-Nordhorn J, Stritter W, Fotopoulou C, Sehouli J, Holmberg C. Symptomization and triggering processes: Ovarian cancer patients' narratives on pre-diagnostic sensation experiences and the initiation of healthcare seeking. Soc Sci Med. 2014;119:123-30.

87. Scott S, McGurk M, Grunfield E. Patient delay for potentially malignant oralsymptoms. Eur J Oral Sci. 2008;116(2):141-7.

88. Scott S, Grunfield E, Auyeung V, McGurk M. Barriers and triggers to seeking help for potentially malignant oral symptoms: implications for interventions. J Public Health Dent. 2009;69(1):34-40.

89. Cooper C, Polonec L, Stewart S, Gelb C. Gynaecologic cancer symptom awareness, concern and care seeking among US women: a multi-site qualitative study. Fam Pract. 2013;30(1):96-104.

90. Lauver D, Coyle M, Panchmatia B. Women's reasons for and barriers to seeking care for breast-cancer symptoms. Womens Health Issues. 1995;5(1):27-35.

91. Cochran S, Hacker N, Berek J. Correlates of delay in seeking treatment for endometrial cancer. J Psychosom Obstet Gynaecol. 1986;5(4):245-52.

92. Corner J, Hopkinson J, Fitzsimmons D, Barclay S, Muers M. Is late diagnosis of lung cancer inevitable? Interview study of patients' recollections of symptoms before diagnosis. Thorax. 2005;60(4):314-9.

93. Gollwitzer P. Goal achievement: The role of intentions. Eur Rev Soc Psychol. 1993:4:441-85

94. Whitaker KL, Winstanley K, Macleod U, Scott SE, Wardle J. Low cancer suspicion following experience of cancer 'warning sign'. Eur J Cancer. 2015 http://dx.doi.org/10.1016/j.ejca.2015.07.014

95. Robb KA, Simon AE, Miles A, Wardle J. Public perceptions of cancer: a qualitative study of the balance of positive and negative beliefs. BMJ Open. 2014:4(7), e005343.

96. Kahneman D. Thinking fast and slow. London: Penguin; 2011.

97. Edwards M, Wood F, Davies M, Edwards A. 'Distributed health literacy': a longitudinal qualitative analysis of the roles of health literacy mediators and social networks of people living with a long term condition. Health Expect. 2013. doi:10.1111/hex.12093

98. Pescosolido B, Boyer C. How do people come to use mental health services? Current knowledge and changing perspectives. In: Scheid T, Brown T, editors. A Handbook for the study of mental health: Social contexts, theories and systems. New York: Cambridge University Press; 1999. p. 392-411.

99. Dobson CM, Russel AJ, Rubin GP. Patient delay in cancer diagnosis: what do we really mean and can we be more specific? BMC Health Serv Res. 2014;14:(387). doi:10.1186/1472-6963-14-387. 